\title{
Spectroscopy and Decays of Charm and Bottom
}

\author{
Joel N. Butler \\ Fermi National Accelerator Laboratory \\ P.O. Box 500, Batavia, Illinois 60510
}

October 1997

Published Proceedings of the VII Mexican School of Particles and Fields and I Latin American Symposium on High Energy Physics, Merida, Yucatan, Mexico, Oct. 3-Nov. 6, 1996 


\section{Disclaimer}

This report was prepared as an account of work sponsored by an agency of the United States Government. Neither the United States Government nor any agency thereof, nor any of their employees, makes any warranty, express or implied, or assumes any legal liability or responsibility for the accuracy, completeness or usefulness of any information, apparatus, product or process disclosed, or represents that its use would not infringe privately owned rights. Reference herein to any specific commercial product, process or service by trade name, trademark, manufacturer or otherwise, does not necessarily constitute or imply its endorsement, recommendation or favoring by the United States Government or any agency thereof. The views and opinions of authors expressed herein do not necessarily state or reflect those of the United States Government or any agency thereof.

\section{Distribution}

Approved for public release: further dissemination unlimited. 


\title{
Spectroscopy and Decays of Charm and Bottom
}

\author{
Joel N. Butler* \\ - Fermi National Accelerator Laboratory ${ }^{1}$ \\ Batavia Ilinois 60510
}

\begin{abstract}
After a brief review of the quark model. we discuss our present knowledge of the spectroscopy of charm and bottom mesons and baryons. We go on to review the lifetimes. semileptonic. and purely leptonic decays of these particles. We conclude with a brief discussion $B$ and $D$ mixing and rare decays.
\end{abstract}

\section{INTRODUCTION}

In the Standard Model(SM) of Elementary Particle Physics, matter is composed of two kinds of particles - quarks and leptons - whose interactions are mediated by vector gauge bosons and who receive their mass via the Higgs mechanism [1].

The quarks undergo all the known kinds of elementary particle interactions: strong, weak, and electromagnetic. While the $u$ and $d$ quarks make up nearly all of ordinary matter, the heavy quarks, charm and bottom (or beauty), are good laboratories for the study of the weak interaction and its interplay with the strong interaction. ${ }^{2}$ They open up a whole field of study of spectroscopy and weak decays that challenges our detailed understanding of particle physics. Hidden within their decays may be examples of important phenomena that fall within the SM, such as CP violation, and possibly new phenomena, such as certain rare decays, which may lie outside the SM and provide us with clues for extending it.

The goal of this paper is to review several topics involving the spectroscopy and weak decay of two flavors of heavy quarks - charm and bottom. For each topic, we show how the Standard Model of elementary particle physics

1) Fermi National Accelerator Laboratory is operated by Universities Research Association under contract to the U.S. Department of Energy.

2) The top quark is so massive that it actually undergoes weak decay before it ever has a chance to form a hadron. This gives it very different behavior from that of charm and bottom. The properties of the top quark are the subject of another lecture at this school. 
adequately accounts for the similarities and differences between these two systems.

We begin with a brief review of the quark model and then discuss the spectroscopy of charm and bottom mesons and baryons, emphasizing excited meson states. We then enter a discussion of weak decays of charm and bottom. We discuss the lifetimes of the various hadrons - what is expected from theory and what has been measured in experiments. Then, we review our knowiedge of semileptonic decays and touch briefly on purely leptonic decays. Finally, we touch on flavor mixing and rare decays within the context of the Standard Model.

\section{A BRIEF REVIEW OF THE QUARK MODEL}

In our current understanding of nature, matter is composed of quarks and leptons. The three 'generations' of quarks and leptons are:

$$
\left(\begin{array}{l}
u \\
d
\end{array}\right)\left(\begin{array}{l}
\nu_{e} \\
e
\end{array}\right) ;\left(\begin{array}{l}
c \\
s
\end{array}\right)\left(\begin{array}{l}
\nu_{\mu} \\
\mu
\end{array}\right) ;\left(\begin{array}{l}
t \\
b
\end{array}\right)\left(\begin{array}{c}
\nu_{\tau} \\
\tau
\end{array}\right) .
$$

- The leptons appear in nature as isolated particles or, in the case of electrons (and muons) can be bound into atoms. The quarks, because of the nature of the color forces which mediate the strong interaction, never appear in isolation but are bound into color singlets. The allowed configurations are

- a quark and an anti-quark - these are the mesons; and

- three quarks (three anti-quarks) - these form the baryons (antibaryons).

Heavy quarks, such as charm and bottom, do not appear in ordinary matter but are produced in high energy collisions. The mesons and baryons which are produced decay via the weak interaction. The decay rate may be estimated by analogy with another weak decay - the decay of the muon - depicted in figure 1 .
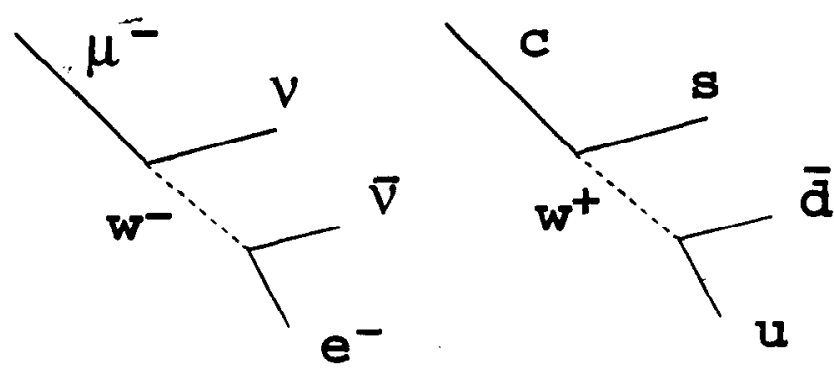

FIGURE 1. Weak Decay of a) muon and b) a charm quark

The rate for muon decay is given by 


$$
\Gamma_{\mu}=\frac{G_{F}^{2}}{192 \pi^{3}} m_{\mu}^{5}
$$

The dependence on the mass to the fifth power is a consequence of phase space and the spin structure of the decay and carries over to the analogous quark decay. The coupling constant, $G_{F}$, must have the unit of (Mass) ${ }^{-2}$.

One expects heavy quarks to decay much more rapidly than muons. The muon's mean lifetime is $2.2 \times 10^{-6} \mathrm{~s}$. The lightest particles containing the charm quark, with a mass of approximately $1.5 \mathrm{GeV} / \mathrm{c}^{2}$, all have mean lifetimes between $1 \times 10^{-12} \mathrm{~s}$ and $1 \times 10^{-13} \mathrm{~s}$. Applying the formula above directly, the ratio of the charm, bottom, and top quark ${ }^{3}$ decay rates should be 1:500:2 $\times$ $10^{10}$.

However, the story is a bit more complicated than this. The coupling 'strength' of the W bosons to quarks and leptons is not required to be the same. It turns out that if we take the strength to be ONE for muons, then it is the same for electrons and $\tau$ 's. However, it is not always the same for the various quarks. The $W$ connects pairs of quarks whose electric charge differs by one unit. For each possible decay there is a 'weak charge' which describes the strength of the W's coupling and we denote these charges by the symbols $V_{q_{2 / 3} q_{1 / 3}}$, where $q_{i}$ represents a quark of electric charge $i$. If the W's connected only the quarks within each generation, then the lightest quark in each generation would be stable. However, this is not what happens. The charge $1 / 3$ quarks may be viewed as 'mixed'. That is, the W couples to linear combinations of the 'physical' quark states - that is the quark states produced in the strong interaction. This gives rise to the so-called CKM [2] matrix which effectively gives the nine coupling strengths of the $W$ bosons to the various combinations of quark decays that it mediates. The CKM matrix is written as:

$$
V=\left(\begin{array}{lll}
V_{u d} & V_{u s} & V_{u b} \\
V_{c d} & V_{c s} & V_{c b} \\
V_{t d} & V_{t s} & V_{t b}
\end{array}\right)
$$

The semileptonic decay width of a heavy quark $Q$ to a light quark $q$ would be,-by way of analogy with muon decay:

$$
\Gamma_{Q \rightarrow q \mu \nu}=\frac{G_{F}^{2}}{192 \pi^{3}} m_{Q}^{5} \times\left|V_{Q q}\right|^{2} \times F(\epsilon)
$$

where $F(\epsilon)$ is a phase space factor with $\epsilon=\frac{M_{q}}{M_{Q}}$.

3) The number for top is not really correct. Because the top is massive enough to decay into a real $\mathrm{W}$ boson, there is only one weak interaction decay vertex and the decay width. by dimensional analysis, depends on the top quark mass to the third power. However, it depends on $G_{F}$ rather than $G_{F}^{2}$ so its decay goes even faster than indicated above. 
The nine CKM matrix elements must form a unitary matrix so the elements are not all independent. Within the SM, the CKM elements are fundamental constants by which we mean there is no prescription for calculating what they are. The determination of the elements of the CKM matrix is a fundamental problem for particle physics and must be done by making measurements of the various properties of weak decays. The methods for determining the CKM elements that involve the heavy quarks are a central theme of this paper. However, we cannot make observations directly upon the quarks themselves since they are always bound into hadrons by the strong interaction. The challenge is to understand the strong interaction well enough to disentangle it from the weak interaction effects so that the CKM elements can be extracted. This situation is depicted in figure 2 which shows a $B$-meson decaying semileptonically to a $D$-meson, electron, and neutrino. It is through study of weak decays and lifetimes that one concludes that $V_{c b}$ is small compared to $V_{c s}$ which gives the $B$-mesons longer lifetimes than charmed mesons even though they are much heavier. In the next section, we will review the status of charm and bottom spectroscopy and we will introduce some ideas which can be used to help deal with the strong interaction effects.

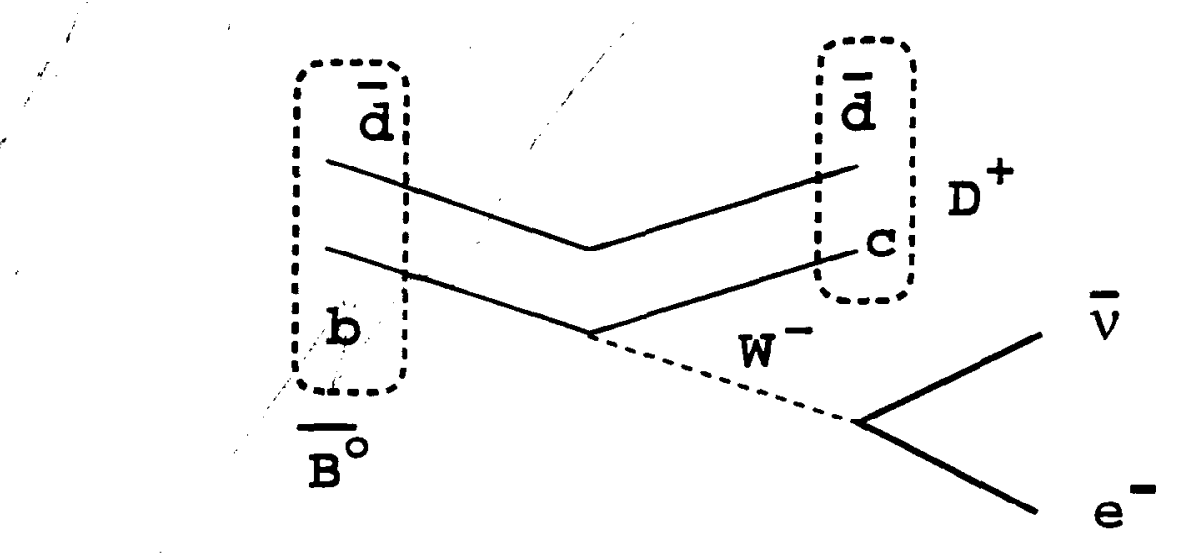

FIGURE 2. Weak semileptonic decay: $\bar{B}^{\circ} \rightarrow D^{+} e^{-} \bar{\nu}$

\section{THE SPECTROSCOPY OF CHARM AND BOTTOM HADRONS}

\section{A Ground-State Charmed Particles}

The charmed mesons, the $D^{\circ}, D^{+}$, and $D_{s}$, are composed of a charmed quark in association with a $\bar{u}, \bar{d}$, and $\bar{s}$ antiquark respectively. These lowest mass mesons are pseudoscalars. The quarks are in a total spin state of 0 and a relative orbital angular momentum state $L=0$. The vector mesons, $D^{* 0}$, 
$D^{*+}$, and $D_{3}^{*+}$, have a total spin of 1 but the charmed quark and light antiquark still have relative angular momentum 0 . The charmed mesons and the light quark mesons form $\mathrm{SU}(4)$ 16-plets shown below in figure 3 .

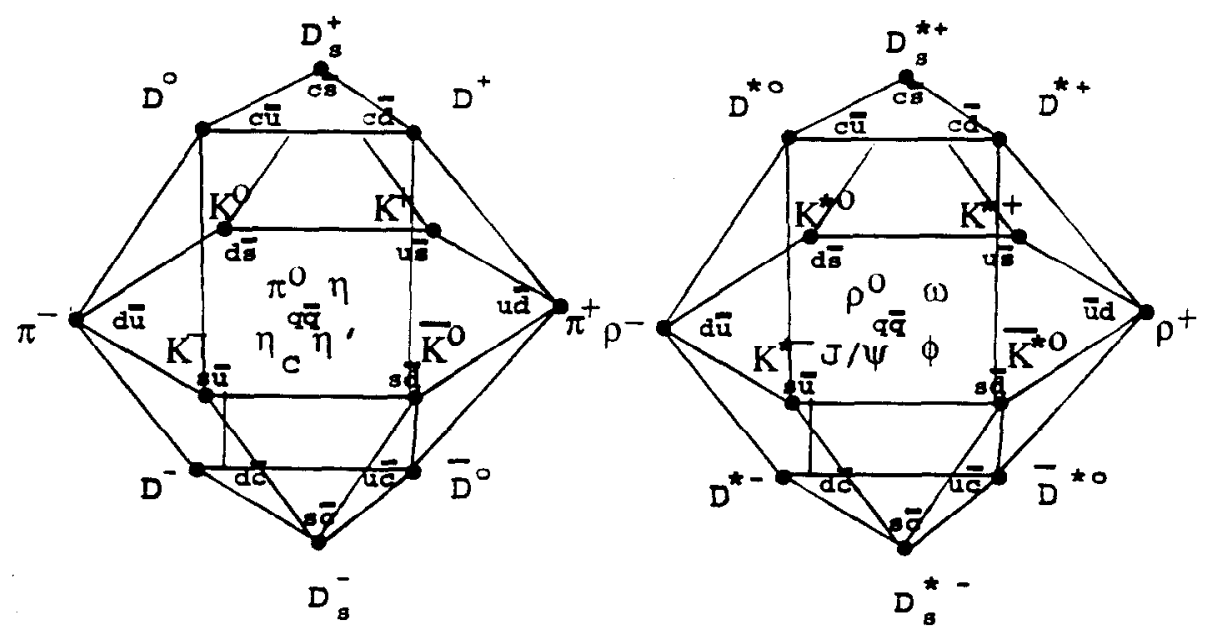

FIGURE 3. SU(4) 16-plets for lowest mass charmed mesons. left side: pseudoscalar mesons; right side: vector mesons. This figure is adapted from reference [3]

The charmed baryons consist of at least one charmed quark and two other quarks. The baryons made of only light quarks obey SU(3) and fall into an octet and a decuplet. When the charmed quark is brought into the picture, $\mathrm{SU}(4)$ symmetry, results in two 20-plets shown below in figure 4.
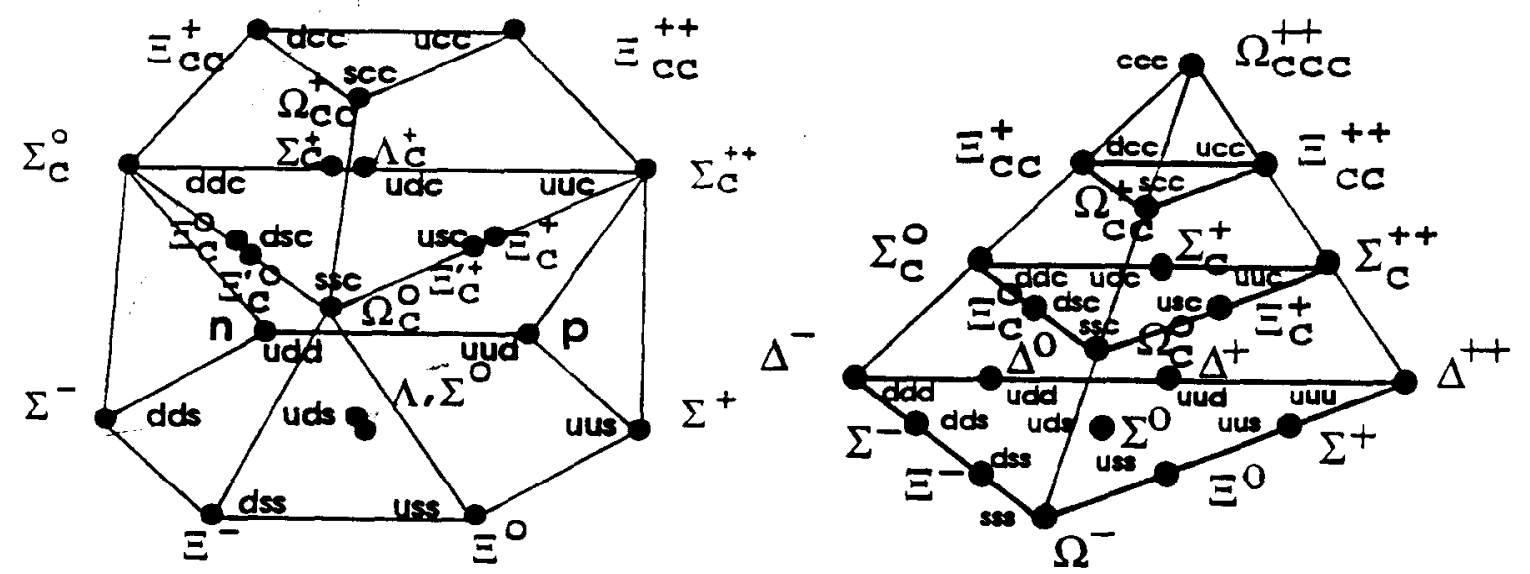

FIGURE 4. SU(4) 20-plets for lowest mass charmed baryons. left side: spin 1/2 baryons; right side: spin $3 / 2$ baryons. This figure is adapted from reference [4].

The nine states on the middle level the spin 1/2 20-plet consist of one charm quark and two light quarks. These can be decomposed into 6 states which are symmetric under interchange of the light quarks and three which 
are antisymmetric. This decompostion is shown in figure 5.
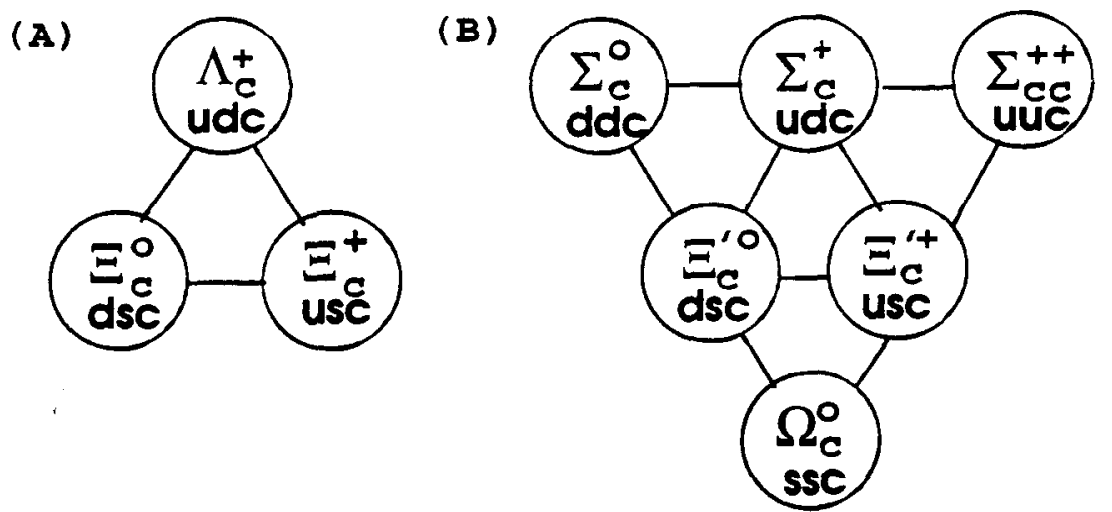

FIGURE 5. Exchange symmetry of the nine ground state spin $1 / 2$ singly charmed baryons: The three states on the left have wavefunctions that are antisymmetric with respect to the interchange of the light quarks; the six on the right are symmetric under interchange. This figure is adapted from reference [5].

The comparable asymmetric states do not exist in the spin 3/2 20-plet because the light quarks must be in a symmetric state to satisfy the requirement that the overall state be antisymmetric. Similarly, the ccc state exists only in the spin $3 / 2$ multiplet.

Not all of the charm baryon states have yet been discovered. In fact, only states with one charmed quark have been convincingly observed and even a few of them have not.yet been observed. Table 1 shows the current experimental situation for these states.

Figure 6 shows the evidence [7] for the $\Omega_{c}$, a baryon whose quark composition is css,decaying into $\Sigma^{+} K^{-} K^{-} \pi^{+}$. The mass of this state is $2700 \mathrm{MeV} / \mathrm{c}^{2}$.

The $\Xi_{c}^{*+}$ decays to $\Xi_{c}^{o} \pi^{+}$. The evidence [8] for this excited state of $\Xi_{c}$ is shown in figure 7.

Figure 8 shows the mass spectra obtained by the CLEO Collaboration [9] for $\Lambda_{c}^{+} \pi^{+}$and $\Lambda_{c}^{+} \pi^{-}$. In each plot, there are two prominent peaks: the one at the lower mass is the $\Sigma_{c}$; and the one at the higher mass is identified as the $\Sigma_{c}^{*}$. The characteristics of the $\Sigma_{c}$ states are

$$
\begin{aligned}
\Delta M\left(\Sigma_{c}^{*++}-\Lambda_{c}^{+}\right) & =234.5 \pm 1.1 \pm 0.8 \mathrm{MeV} \\
\Gamma\left(\Sigma_{c}^{*++}\right) & =17.9_{-3.2}^{+3.8} \pm 4.0 \mathrm{MeV} \\
\Delta M\left(\Sigma_{c}^{* 0}-\Lambda_{c}^{+}\right) & =232.6 \pm 1.0 \pm 0.8 \mathrm{MeV} \\
\Gamma\left(\Sigma_{c}^{* 0}\right) & =13.0_{-3.0}^{+3.7} \pm 4.0 \mathrm{MeV} \\
\Delta M\left(\Sigma_{c}^{*++}-\Sigma_{c}^{* 0}\right) & =1.9 \pm 1.4 \pm 1.0 \mathrm{MeV}
\end{aligned}
$$

By analogy with atomic physics, we also expect excited states of the charmed mesons and baryons which have angular momentum between the quarks or feature higher radial excitations. These will be discussed in detail 
TABLE 1. Charmed $1 / 2^{+}$baryon states. $[a, b]$ and $\{a, b\}$ denote antisymmetric and symmetric flavor index combinations. This table is adapted from reference [6]. Values in parentheses on states labelled 'not seen' are taken from this reference and are included to gently guide searches for them.

\begin{tabular}{|c|c|c|c|c|c|c|}
\hline Notation & $\begin{array}{c}\text { Quark } \\
\text { content }\end{array}$ & $\mathrm{SU}(3)$ & $\left(I, I_{3}\right)$ & $\mathrm{S}$ & $\mathrm{C}$ & $\begin{array}{c}\text { Mass } \\
(\mathrm{MeV})\end{array}$ \\
\hline$I_{c}^{+}$ & $c[u d]$ & $3^{*}$ & $(0,0)$ & 0 & 1 & $2285.1 \pm 0.6$ \\
\hline$\Xi_{\varepsilon}^{+}$ & $c[s u]$ & $3^{*}$ & $(1 / 2,1 / 2)$ & -1 & 1 & $2465.1 \pm 1.6$ \\
\hline$\Xi:$ & $c[s d]$ & $3^{*}$ & $(1 / 2,-1 / 2)$ & -1 & 1 & $2470.3 \pm 1.8$ \\
\hline$\Sigma_{\varepsilon}^{++}$ & cนu & 6 & $(1,1)$ & 0 & 1 & $2453.1 \pm 0.6$ \\
\hline$\Sigma_{c}^{+}$ & $c\{u d\}$ & 6 & $(1,0)$ & 0 & 1 & $2453.8 \pm 0.9$ \\
\hline$\Sigma_{c}^{0}$ & cdd & 6 & $(1,-1)$ & 0 & 1 & $2452.4 \pm 0.7$ \\
\hline$\Xi_{\varepsilon}^{+}$ & $c\{s u\}$ & 6 & $(1 / 2,1 / 2)$ & -1 & 1 & not $\operatorname{seen}(2561)$ \\
\hline$\Xi_{e}^{o^{\prime}}$ & $c\{s d\}$ & 6 & $(1 / 2,-1 / 2)$ & -1 & 1 & not seen $(2561)$ \\
\hline$\Omega_{0}^{0}$ & css & 6 & $(0,0)$ & -2 & 1 & $2705.0 \pm 1.0$ \\
\hline$\Xi_{c e}^{++}$ & cces & 3 & $(1 / 2,1 / 2)$ & 0 & 2 & not seen $(3616)$ \\
\hline$\Xi_{e c}^{+}$ & $\operatorname{ccd}$ & 3 & $(1 / 2,-1 / 2)$ & 0 & 2 & not seen $(3616)$ \\
\hline$\Omega_{c c}^{+}$ & $\operatorname{ccs}$ & 3 & $(0,0)$ & -1 & 2 & not seen $(3706)$ \\
\hline
\end{tabular}

later. However, to round out our tour of the charmed baryons, we anticipate this discussion by showing candidates for such excited baryon states, the $\Lambda_{c}^{*+}(2593)$ and $\Lambda_{c}^{*+}(2630)$ [10]. These appear as resonances in the invariant mass spectra $\Lambda_{c}^{+} \pi^{+} \pi^{-}$shown in figure 9 . Two peaks are seen. These states are interpreted as consisting of an $L=0, S=0$ light diquark orbiting around a $c$-quark with relative orbital angular momentum $L$ equal to 1 . This state is shown schematically in figure 10. The lighter state is identified with total angular momentum $J=1 / 2$ state and the heavier with $J=3 / 2$. The light one decays through $\Sigma_{c} \pi$ but the heavier one cannot decay to its favored mode $\Sigma_{c}^{*} \pi$ which is too massive so it goes through a decay to a virtual $\Sigma_{c}^{*} \pi$ state with the virtual $\Sigma_{c}^{*}$ decaying to $\Lambda_{c} \pi$.

\section{B Ground State Bottom Particles}

The ground state $B$-meson family is very similar to the $D$-meson family and consists of three $S=0$ and three $S=1$ states:

$$
\begin{gathered}
B^{\circ}, B^{+}, B_{s}^{\circ} \\
B^{* 0}, B^{*+}, B^{* 0}
\end{gathered}
$$

All these states have been observed. In addition, there is the as yet unobserved $B_{c}$, a state consisting of a $\bar{b}$ quark and a charm quark. This state is truly fascinating because it is a flavor-bearing state consisting of two heavy quarks. Nonrelativistic potential models should provide an excellent description of its 
TABLE 2. Charmed $3 / 2^{+}$baryon states. This table is adapted from reference [6]. Values in parentheses on states labelled 'not seen' are taken from this reference and are included to gently guide searches for them. For observed states. mass values are from PDG or. if not listed. my estimates ( $\Xi_{c}^{*}$ 's and $\Sigma_{c}^{*}$ 's).

\begin{tabular}{||c|c|c|c|c|c|c||}
\hline Notation & $\begin{array}{c}\text { Quark } \\
\text { content }\end{array}$ & $\mathrm{SU}(3)$ & $\left(I, I_{3}\right)$ & $S$ & $\mathrm{C}$ & $\begin{array}{c}\text { Mass } \\
(\mathrm{MeV})\end{array}$ \\
\hline$\Sigma_{c}^{*++}$ & cur & 6 & $(1,1)$ & 0 & 1 & $2519.6 \pm 2$ \\
$\Sigma_{c}^{*+}$ & cud & 6 & $(1,0)$ & 0 & 1 & not seen(2545) \\
$\Sigma_{c}^{* *}$ & cdd & 6 & $(1,-1)$ & 0 & 1 & $2517.7 \pm 2$ \\
$\Xi_{c}^{*+}$ & cus & 6 & $(1 / 2,1 / 2)$ & -1 & 1 & $2645 \pm 2$ \\
$\Xi_{c}^{* *}$ & $c d s$ & 6 & $(1 / 2,-1 / 2)$ & -1 & 1 & $2644 \pm 2$ \\
$\Omega_{c}^{* *}$ & $c s s$ & 6 & $(0,0)$ & -2 & 1 & not seen(2778) \\
\hline$\Xi_{c c}^{*++}$ & $c c u$ & 3 & $(1 / 2,1 / 2)$ & 0 & 2 & not seen(3744) \\
$\Xi_{c c}^{*+}$ & $c c d$ & 3 & $(1 / 2,-1 / 2)$ & 0 & 2 & not seen(3744) \\
$\Omega_{c c}^{*+}$ & $c c s$ & 3 & $(0,0)$ & -1 & 2 & not seen(3838) \\
\hline$\Omega_{c e c}^{*+}$ & $c c c$ & 1 & $(0,0)$ & 0 & 3 & not seen(4797) \\
\hline
\end{tabular}

wave function. A very rich collection of excited states exist and they decay by radiative and hadronic transitions. A study of these would provide insight into the interquark potential when the quarks are very close to being at rest. The spectroscopy of the $B_{\varepsilon}$ 'state is discussed below.

The mass separation between the $B^{* \prime}$ s and the $B$ 's is smaller than between the $D^{*}$ 's and $D^{\prime}$ 's: about $45 \mathrm{MeV} / \mathrm{c}^{2}$ as compared to $145 \mathrm{MeV} / \mathrm{c}^{2}$. The reason, which will be discussed more later on, is due to the fact that the hyperfine splitting, which is responsible for the difference between the $S=0$ and $S=1$ states, goes like $\frac{1}{M_{Q}}$ where $M_{Q}$ is the mass of the heavy quark. Because of this, all $B^{*}$ 's are below threshold for decay through $B \pi$ and decay radiatively:

$$
B^{*} \rightarrow B \gamma
$$

The $b$-baryon family is quite large. Very little is known about $b$-baryons except for the $\Lambda_{b}$ which has been observed through its decay to $\psi \Lambda$ and its semileptonic decay to $\Lambda_{c}$. Figure 11 shows the $\Lambda_{b}$ signal obtained by the CDF experiment at Fermilab [11]. The mass values obtained by various experiments [12] are given in table 3 .

TABLE 3. Mass values for $\Lambda_{b}$.

\begin{tabular}{||c|c||}
\hline Exp. & Mass (MeV/c $\left.{ }^{2}\right)$ \\
\hline CDF & $5621 \pm 4$ (stat) \pm 3 (syst) \\
ALEPH & $5614 \pm 21$ (stat) \pm 4 (syst) \\
DELPHI & $5668 \pm 16$ (stat) \pm 8 (syst) \\
UA1 & $5640 \pm 50$ (stat) \pm 30 (syst) \\
\hline
\end{tabular}



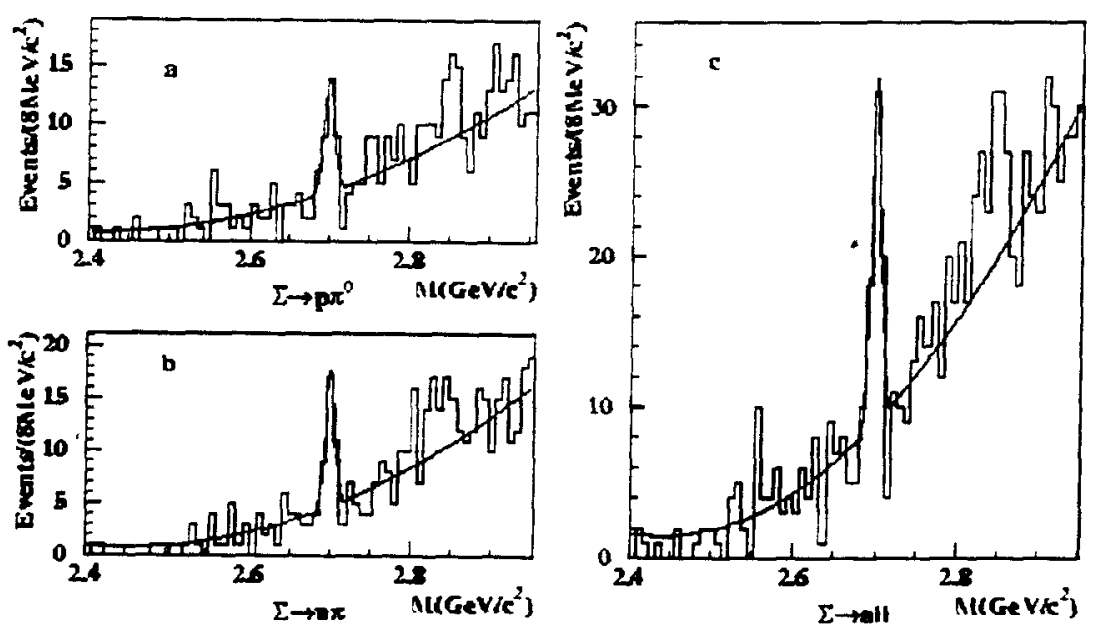

FIGURE 6. Invariant mass spectrum for the final state $\Sigma^{+} K^{-} K^{-} \pi^{+}$, showing a narrow peak around $2700 \mathrm{MeV} / \mathrm{c}$ from Fermilab experiment 687. a) shows the spectrum when the $\Sigma$ decays through $p \pi^{\circ}$; b) shows the spectrum when the $\Sigma$ decays through $n \pi^{+}:$and $c$ ) is the combined spectrum.

\section{The Physics of Excited States of Charm and Bottom}

In addition to the states we have discussed so far, there are also states with orbital angular momentum between the quarks. These states are often referred to as generically $D^{* *}$ 's and $B^{* * \prime} s$. The existence of these states is established for both charm and bottom. Taken together, the spectra of these states permits some important tests of key ideas about heavy quark states and how the light degrees of freedom behave in these states. These ideas carry over to the discussion of how the hadronic degrees of freedom influence the extraction of weak interaction parameters in the study of the semileptonic decays of hadrons.

Excited $D$ mesons have higher values of the radial or orbital quantum number and have higher mass. The first such excited state was seen by the ARGUS collaboration in 1986 [13]. Since then, 5 additional excited charmed meson states have been seen [14]. The main contributors to the experimental observations have been the ARGUS experiment, CLEO, and Fermilab experiments E691 and E687. LEP experiments and Fermilab Experiment 791 are also beginning to contribute to our knowledge of these states.

The first set of excited states are expected to have $L=1$, shown schematically in figure 12. These states all have positive parity. Since the spin of the light quark, $\vec{s}_{q=u, d, s}$, and that of the heavy quark, $\vec{s}_{Q=c, b}$, can add to a 


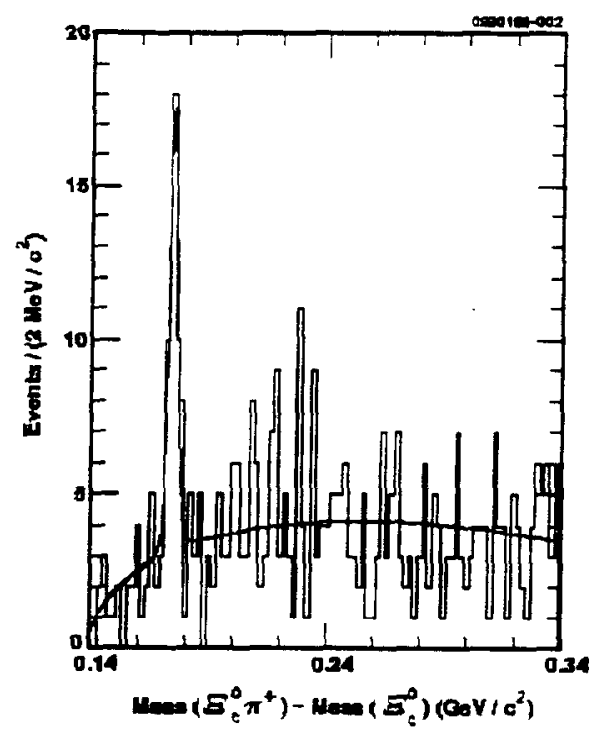

FIGURE 7. Invariant mass spectrum for the final state $\Xi_{c}^{0} \pi^{+}$, showing a narrow peak about $174 \mathrm{MeV} / \mathrm{c}^{2}$ above the $\Xi_{c}$ from CLEO.

total spin $\vec{S}$ of 0 or 1 , the total angular momentum, $J$, can be 0,1 , or 2 .

Assuming that these states are heavy enough to decay strongly into a ground state charmed meson and a pseudoscalar meson ( $\pi$ or K), then conservation rules such as angular momentum, parity, and isospin lead to twelve states with the pattern of quantum numbers and allowed decays shown here ${ }^{4}$ :

TABLE 4. Quantum numbers and allowed decays of excited charmed mesons, using total quark spin, orbital angular momentum, and total angular momentum to describe the states.

\begin{tabular}{||c|c|c|c||}
\hline${ }^{25+1} L_{J}$ & $c \bar{u}$ & $c d$ & $c \bar{s}$ \\
\hline${ }^{-3} P_{2}$ & $D \pi, D^{*} \pi$ & $D \pi, D^{*} \pi$ & $D^{*} K, D K$ \\
${ }^{3} P_{1}$ & $D^{*} \pi$ & $D^{*} \pi$ & $D^{*} K$ \\
${ }^{3} P_{0}$ & $D \pi$ & $D \pi$ & $D K$ \\
${ }^{1} P_{1}$ & $D^{*} \pi$ & $D^{*} \pi$ & $D^{*} K$ \\
\hline
\end{tabular}

While these quantum numbers may be an 'appropriate choice' for charmonium where the masses of the two quarks are equal, they may not be 'appropriate' to a 'heavy-light' system. By 'appropriate choice' we mean that the quantum numbers best express the symmetry of the system (leaving the rest

4) One complication is that the two $1^{+}$states can mix. We shall ignore this in the following discussion. 
$=2.0$

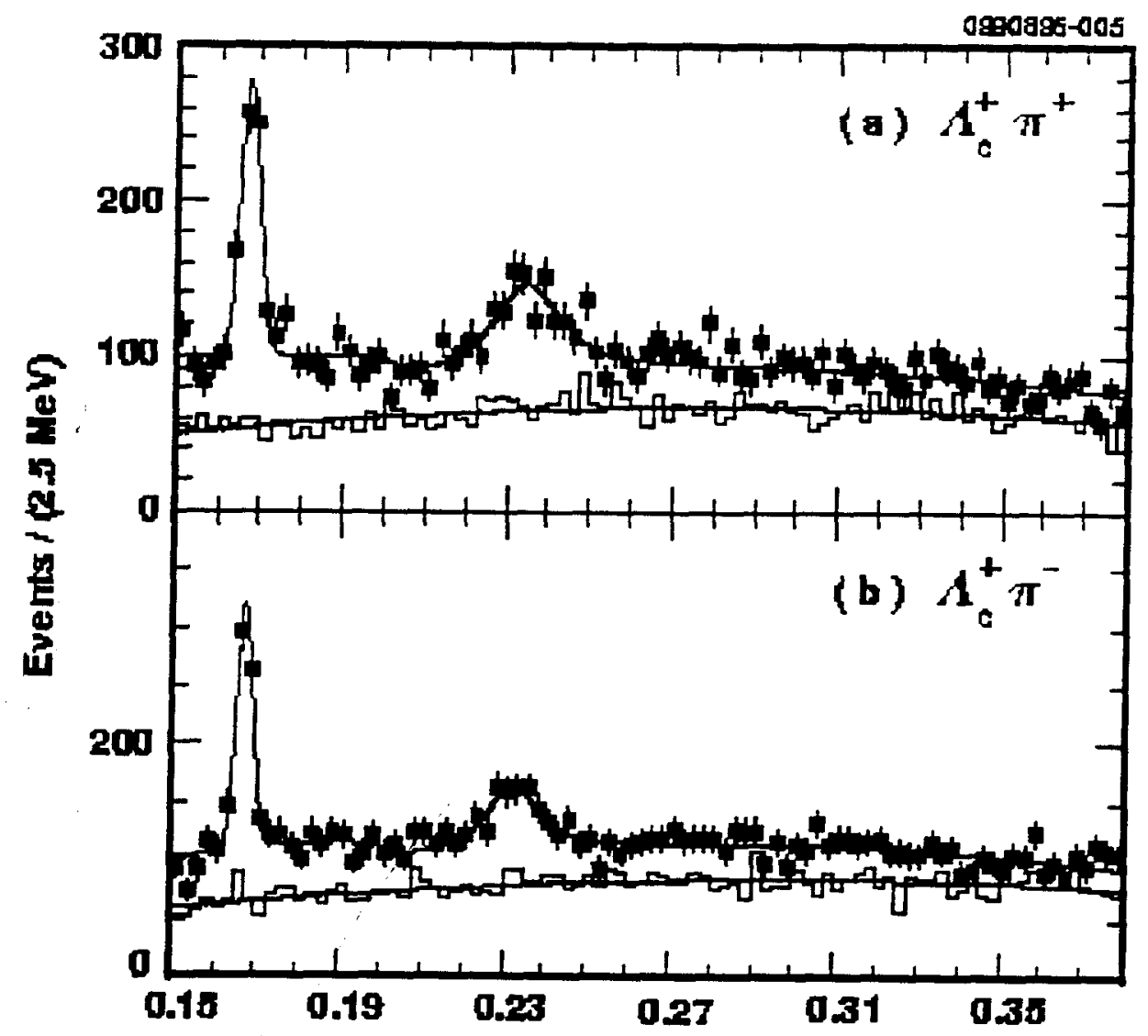

FIGURE 8. Invariant mass spectrum for the final states $\Lambda_{c}^{+} \pi^{+}$(upper), and $\Lambda_{c}^{+} \pi^{-}$(lower) from CLEO.

as a small perturbation). For example, in the hydrogen atom, we do not worry about the nuclear spin - it largely decouples from the spectroscopy and enters only as a 'hyperfine' effect.

One very relevant symmetry is 'Heavy Quark Symmetry' or HQS which is supported by a 'Heavy Quark Effective Theory' or HQET [15]. According to this, in the limit of infinitely heavy quark mass, the heavy and light degrees of freedom decouple and the light degrees of freedom determine the quantum states, level spacing, and decay rates (and hence widths) of the heavy-light mesons. This same approach leads to relations between the transition matrix elements which appear, for example, in semileptonic decays of these systems.

The model is expected to be a good approximation when

$$
M_{Q}>\Lambda_{Q C D}
$$

and, therefore should apply to the $b$-quark and hopefully the c-quark. Corrections would be expected to be of order $\frac{\Lambda_{Q C D}}{M_{Q}}$ for these finite mass quarks. 


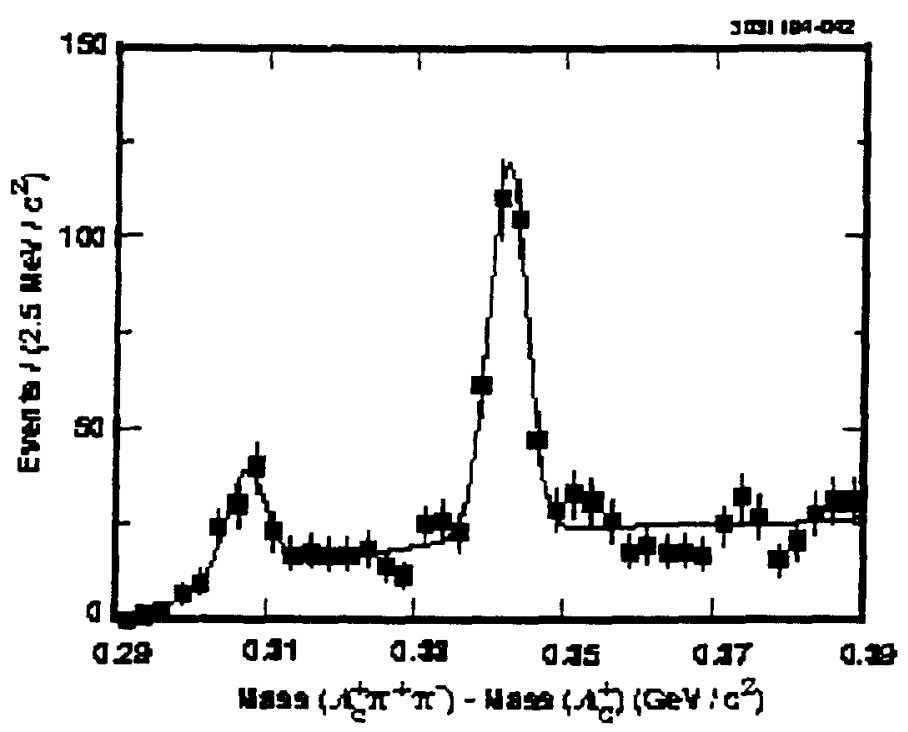

FIGURE 9. Invariant mass spectrum for the final states $\Lambda_{c}^{+} \pi^{+} \pi^{-}$from CLEO.

'In this picture, the best choice for the quantum numbers would be the spin of the heavy quark,

$$
\vec{S}_{Q}
$$

and the total angular momentum of the light degrees of freedom:

$$
\vec{j}=\vec{L}_{\bar{q}}+\overrightarrow{s_{\bar{q}}}
$$

According to Heavy Quark Symmetry, the quantum number $j$ and the quantum number $S_{Q}$ are separately conserved and the static properties and decay rates of the particles will depend only on the light degrees of freedom. For any value of $L$ there are two values of the angular momentum of the light degrees of freedom: $j=L \pm \frac{1}{2}$. For $L=1$, this gives two sets of levels: $j=\frac{3}{2}$ and $j=\frac{1}{2}$. In the heavy quark limit, each level consists of two degenerate states corresponding to the different orientations of the heavy quark spin. For finite $(c, b)$ quark masses, the degeneracy will be broken to the order of $\frac{\Lambda_{Q C D}}{M_{b, e}}$. The $j=\frac{3}{2}, 2^{+}$state decays through a $D$-wave so it is expected to be relatively narrow. Because the quantum numbers of heavy and light degrees of freedom are independently conserved, $j=\frac{3}{2}, 1^{+}$state also decays by a $D$-wave. This means that HQET predicts that the $j=\frac{3}{2}$ states are relatively narrow. Similarly, the $j=\frac{1}{2}, 0^{+}$state must decay via an $\mathrm{S}$-wave and therefore is expected to be very broad - with widths of $100 \mathrm{MeV} / \mathrm{c}^{2}$ or more. Because the quantum numbers of heavy and light degrees of freedom are independently conserved, HQET predicts that the $j=\frac{1}{2}, 1^{+}$state decays purely by S-wave also. Taken together, this means that the $j=\frac{3}{2}$ states are relatively narrow while the 


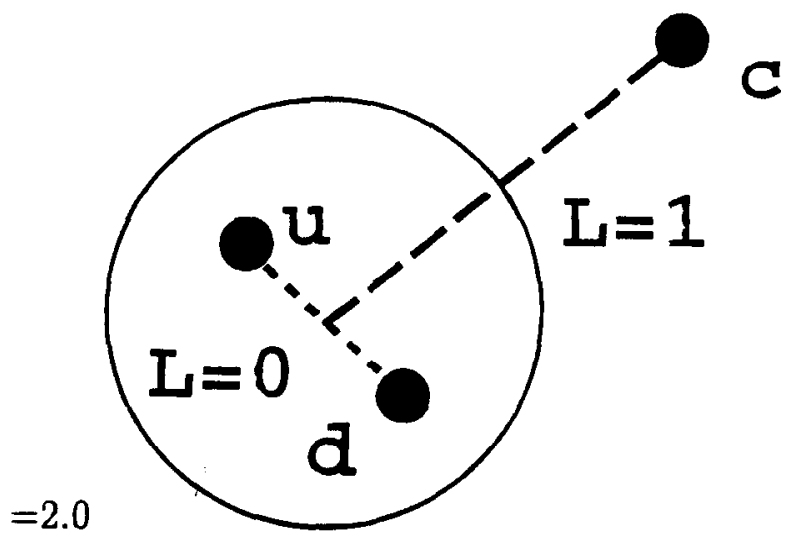

FIGURE 10. Schematic representation of quarks forming $\Lambda_{c}^{*}$ states.

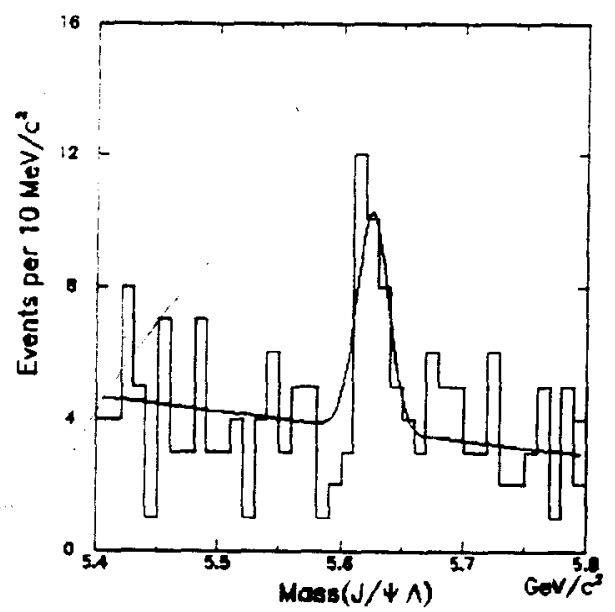

FIGURE 11. Invariant mass spectrum of $\psi \Lambda^{\circ}$ obtained by CDF experiment

$j=\frac{1}{2}$ states are quite broad. Experimental backgrounds make the identification of broad states very difficult and none has so far been convincingly observed. The rest of this paper will confine its attention to the $j=\frac{3}{2}$ states. To differentiate the two $j=\frac{3}{2}$ states, we refer to them as the $1^{+}$and the $2^{+}$ from now on. The predicted spectrum is shown in figure 13.

HQS predicts specific relationships between the level spacing of the $D^{* *}$ 's and $B^{* * \prime}$ s. Symmetry breaking effects in the Hamiltonian can be used to predict the splitting of the $3 / 2$ and $1 / 2$ states. Attempts have even been made to extend the applicability of the symmetry to strange mesons.

Quantum number restrictions similar to the ones shown above also exist. The non-strange $D^{* *} 2^{+}$state can decay into $D^{*} \pi$ or $D \pi$. The $1^{+}$only decays through $D^{*} \pi$. Similarly, the $D_{*}^{* *} 2^{+}$state can decay into $D^{*} K$ or $D K$ while the $1^{+}$state can only decay through $D^{*} K$. In addition, the model makes specific predictions on the decay rates into charm particles and light mesons. 


\section{4}

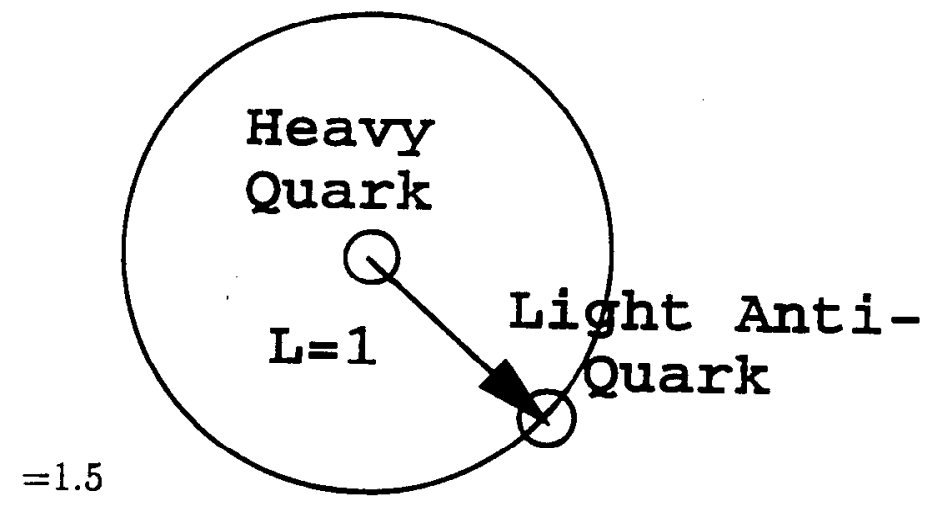

FIGURE 12. Schematic representation of an excited $D$ meson

\section{PDG}

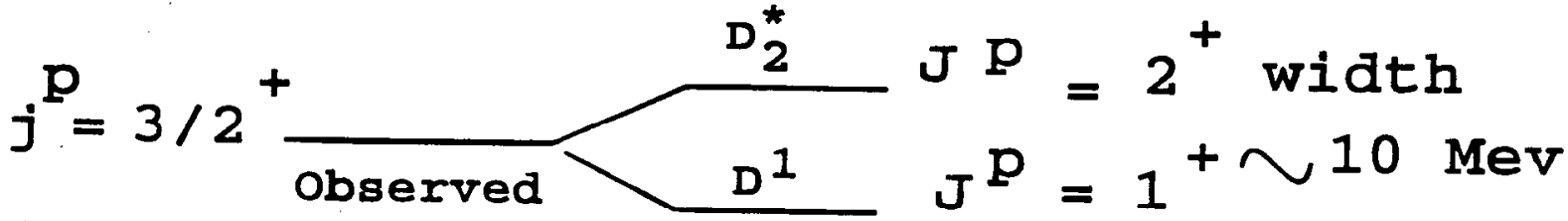

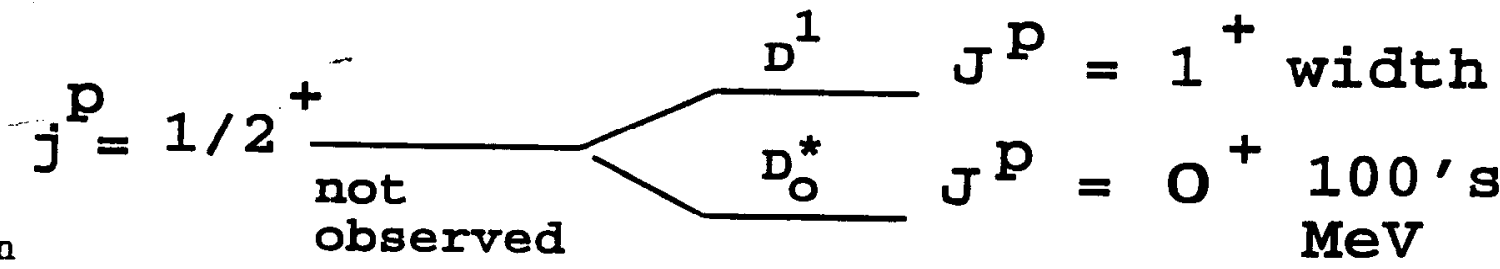

FIGURE 13. Level diagram for $L=1$ excited mesons according to Heavy Quark Symmetry 
HQS also predicts that the decay rates from each excited state into each final state, such as $D \pi, D^{*} \pi, D \rho$, and $D \eta$, are independent of the heavy quark mass.

Using HQS and some arguments about the hadronic form factors, it is possible to estimate the widths of the various excited mesons into various final states and to add them up to get estimates of the total widths. Pionic transitions between any two heavy-light states should be identical independent of the heavy quark mass so charm transitions can be used to predict $B$ transitions. One prediction is that $\frac{\Gamma\left(D_{2}^{*} \rightarrow D \pi\right)}{\Gamma\left(D_{2}^{*} \rightarrow D^{*} \pi\right)}$ is about 1.8 .

If one accepts the validity of the model, then relative rates into different final states, total widths, and certain angular correlations can help associate particular mass bumps with particular states predicted by the HQS model.

\section{Experimental Results on Excited Charm States}

Figure 14 shows the invariant mass difference distributions $M_{D^{+} \pi^{-}}-M_{D^{+}}$ and $M_{D^{\circ} \pi^{+}}-M_{D^{\circ}}$ from $E 687$ [16]. (It is conventional in searches for $D^{* * 1} \mathrm{~s}$ to show the mass difference distribution since certain systematic errors are cancelled on an event-by-event basis.) There are clear peaks near $600 \mathrm{MeV} / \mathrm{c}^{2}$. These distributions are obtained by taking $D$-meson decays into simple final states and combining them with pions of the appropriate sign that emerge from the primary interaction vertex. There is a lot more background in such plots than typically occurs in the spectra of weakly decaying mesons. This is because of the unavoidable background that comes from combining the real $D$ signal (in exeriments where the $D$ moves in the $\mathrm{lab}$ and a vertex detector is available, like E687, the states forming the $D$ signal can be required to be wellseparated from the interaction vertex so that there is little background to the $D$-meson itself) with pions coming from the underlying hadronic interaction and having nothing to do with the decay of a strong resonance. The generation of this 'combinatoric background' is shown schematically in figure 15. The high levels of background make it difficult to isolate the 'broad' states predicted by the theory.

The identification of various peaks with particular quantum states is based on model calculations of the masses, widths, and decay rates. However, in some cases, it is actually possible to extract the quantum numbers of the state. This is true when the state can decay into a $D^{*+}$ and a $\pi$, since the angular distribution between the daughter pion and the pion from the decay of the $D^{*}$ in the $D^{*}$ rest frame conveys information on the overall angular momentum. For more details on these very beautiful analyses, see reference [18].

Tables 5 and 6 [17] summarize the present knowledge of the masses and widths of the six $j=\frac{3}{2}$ excited mesons which have been observed. While the overall picture is satisfactory, there are differences in the mass values that lie outside of the quoted statistical and systematic uncertainties. I attribute 

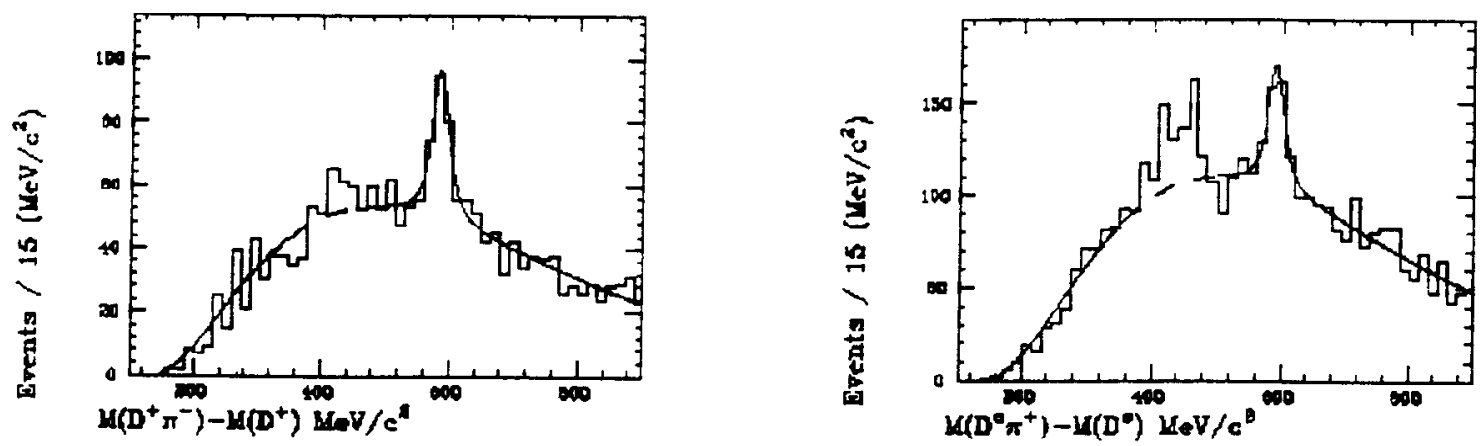

FIGURE 14. Examples of signais for $D^{* *}$ mesons from E687. Left side: Invariant mass difference distribution, $M\left(D^{+} \pi^{-}\right)-M\left(D^{+}\right)$showing a peak around $600 \mathrm{MeV} / \mathrm{c}^{2}$, or a total mass of about $2460 \mathrm{MeV} / \mathrm{c}^{2}$; and right side: Invariant mass difference distribution, $M\left(D^{\circ} \pi^{+}\right)-M\left(D^{\circ}\right)$ showing a peak at almost the same mass. The peak near $400 \mathrm{MeV} / \mathrm{c}^{2}$ is believed to be a feed down from the $D^{* 0} \pi^{+}$decay mode of the same state. where the $D^{\circ *} \hookrightarrow D^{\circ} \pi^{\circ}$ since the $\pi^{\circ}$ is not reconstructed in this analysis. These two plots are identified as the two isospin partners of the $2^{+}$excited $D$-mesons.

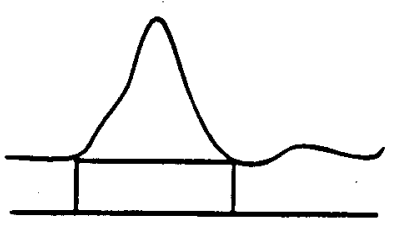

Mass Plot -

-.. shaded area

1s source of background
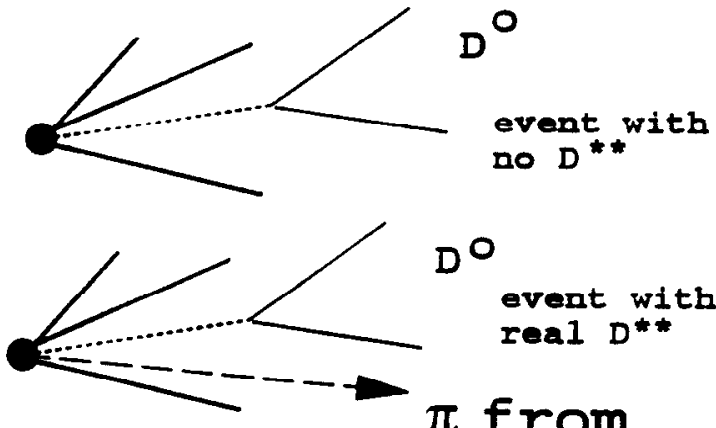

$D^{\circ}$

event with real $D^{* *}$

\section{$\pi$ from}

$\mathrm{D}$

FIGURE 15. Mechanisms for background generation in searches for excited mesons. left side: depicts background faking the $D$, which can combine with $\pi$ 's from the primary to generate background to the $D^{* *}$; right side upper: an event with a real $D$ but no $D^{* *}$ can produce background combinations with $\pi$ 's coming from the primary vertex; and lower right side: a real $D^{* *}$. 
TABLE 5. Properties of the $2^{+}$States

\begin{tabular}{|c|c|c|}
\hline \hline experiment & Mass $\left(\mathrm{MeV} / \mathrm{c}^{2}\right)$ & Width $\left(\mathrm{MeV} / \mathrm{c}^{2}\right)$ \\
\hline$D^{* * 0} \rightarrow D^{+} \pi^{-}:$ \\
\hline E687 & $2453 \pm 3 \pm 2$ & $25 \pm 10 \pm 5$ \\
E691 & $2459 \pm 3 \pm 2$ & $20 \pm 10 \pm 5$ \\
ARGUS & $2455 \pm 3 \pm 5$ & $15_{-10+10}^{+13+5}$ \\
CLEO 1.5 & $2461 \pm 3 \pm 1$ & $20_{-12-10}^{+9+9}$ \\
CLEO II & $2465 \pm 3 \pm 3$ & $28_{-7}^{+8} \pm 6$ \\
\hline$D^{* *+} \rightarrow D^{\circ} \pi^{+}:$ \\
\hline E687 & $2453 \pm 3 \pm 2$ & $23 \pm 9 \pm 5$ \\
ARGUS & $2469 \pm 4 \pm 6$ & $27 \pm 12$ \\
CLEO II & $2463 \pm 3 \pm 3$ & $27_{-8}^{+11} \pm 5$ \\
\hline$D_{3}^{* *+} \rightarrow D^{\circ} K^{+}:$ \\
\hline CLEO II & $2573.3_{-1.6}^{+1.7} \pm 0.9$ & $16_{-4}^{+6} \pm 3$ \\
\hline
\end{tabular}

TABLE 6. Properties of the $1^{+}$States

\begin{tabular}{|c|c|c|}
\hline \hline experiment & Mass $\left(\mathrm{MeV} / \mathrm{c}^{2}\right)$ & Width $\left(\mathrm{MeV} / \mathrm{c}^{2}\right)$ \\
\hline$D^{* * 0} \rightarrow D^{*+} \pi^{-}:$ \\
\hline ARGUS & $2414 \pm 2 \pm 5$ & $13_{-6-5}^{+6+10}$ \\
CLEO 1.5 & $2428 \pm 3 \pm 2$ & $23_{-6-4}^{+8+10}$ \\
CLEO II & $2421 \pm 2 \pm 2$ & $20_{-5-3}^{+6+3}$ \\
E687 & $2422 \pm 2 \pm 2$ & $15^{+6} \pm 8 \pm 4$ \\
\hline$D^{* *+} \rightarrow D^{* 0} \pi^{+}:$ \\
\hline CLEO II & $2425 \pm 2 \pm 2$ & $26_{-7}^{+8} \pm 4$ \\
\hline$D_{r}^{*+*} \rightarrow D^{\circ 0} K^{+}:$ \\
\hline ARGUS & $2535.5 \pm 0.4 \pm 1.3$ & $<3.9(90 \% \mathrm{CL})$ \\
CLEO II & $2535.1 \pm 0.2 \pm 0.5$ & $<2.3(90 \% \mathrm{CL})$ \\
E687 & $2535.0 \pm 0.6 \pm 1.0$ & $<3.2(90 \% \mathrm{CL})$ \\
\hline
\end{tabular}

this to the large and highly structured backgrounds. The structures limit the region over which fits can be performed. In some cases, subtle variations of the fit near the signal region can cause shifts in the mass values. In general, I feel that the quoted systematic errors tend to be optimistic.

\section{E Prediction of Excited B-meson Spectra from Charm Meson Spectra Using an HQS-motivated Approach}

Eichten, Quigg, and Hill [19] write the equations for the mass difference between the ground state and $j=3 / 2$ excited state strange and charmed mesons as: 
TABLE 7. Masses (in $\mathrm{MeV}$ ) predicted for the $2 P\left(\frac{3}{2}\right)$ levels of the $B, D_{s}$, and $B_{s}$ systems. Underlined entries are spin-averaged quantities (derived from Particle Data Group masses) used as inputs.

\begin{tabular}{|c|c|c|c|c|c|}
\hline Meson Family & $\overline{\mathrm{K}}$ & $\bar{D}$ & $\overline{B B}$ & $\overline{D_{s}}$ & $\bar{B}$ \\
\hline $\bar{M}(1 \mathrm{~S})$ & 794.3 & 1973.2 & 0313.1 & 2074.9 & 5403.0 \\
\hline $\bar{M}\left(2^{+}\left(\frac{3}{2}\right)\right)$ & $1429 \pm 6$ & $2459.4 \pm 2.2$ & 5771 & 2561 & 5861 \\
\hline$M\left(1^{+}\left(\frac{3}{2}\right)\right)$ & $1270 \pm 10$ & $2424 \pm 6$ & 5759 & 2526 & 5849 \\
\hline$M\left(2^{+}\left(\frac{3}{2}\right)\right)-M\left(1^{+}\left(\frac{3}{2}\right)\right)$ & 159 & 35 & 12 & 35 & 12 \\
\hline
\end{tabular}

$$
\begin{aligned}
& M\left(2 P_{2}\right)_{K}-M(1 S)_{K}=E(2 P)_{K}+\frac{C\left(2 P_{2}\right)}{m_{s}} \\
& M\left(2 P_{1}\right)_{K}-M(1 S)_{K}=E(2 P)_{K}+\frac{C\left(2 P_{1}\right)}{m_{s}} \\
& M\left(2 P_{2}\right)_{D}-M(1 S)_{D}=E(2 P)_{D}+\frac{C\left(2 P_{2}\right)}{m_{c}} \\
& M\left(2 P_{1}\right)_{D}-M(1 S)_{D}=E(2 P)_{D}+\frac{C\left(2 P_{1}\right)}{m_{c}}
\end{aligned}
$$

For the masses and levels, the notation is $n L_{J}$, where $n$ is the principal quantum number. Here, $M(1 S)=\left[3 M\left(1 S_{1}\right)+M\left(1 S_{0}\right)\right] / 4$ is the spin-averaged mass of the ground state. The difference $E(2 P)_{K}-E(2 P)_{D}$ is determined by potential models to be $32 \mathrm{MeV}$. This leaves 5 unknowns: $E(2 P)_{D}, C\left(2 P_{2}\right)$, $C\left(2 P_{1}\right)$, and $m$, and $m_{c}$. The charm mass was fixed at various values and particular states were used to determine the mass splittings on the left side of the equations. This leaves four equations in four unknowns.

The parameters so determined are used to predict the $j=\frac{3}{2}$ excited $B$ meson states. The results are given in table 7 [19].

\section{F Experimental Results on Excited B-Mesons}

The predicted excited states of the $B$ (non-strange) mesons are listed in table 8. In this table, we use notation motivated by HQS and also use the names which the PDG has adopted for these states.

The $B^{* *}$ states were first observed by LEP experiments. Figure 16 below shows the signal for $B^{* *}$ 's from the ALEPH experiment [20]. The analysis is done by identifying $B$-jets produced in $Z$ decays using the ALEPH vertex detector and then combining the jet momentum vector with that of pions not associated with the other $B$-jet. The analysis cannot distinguish between a $B$ and a $B^{*}$ so the notation $B^{(*)}$ is used to denote a state which could contain either. The masses of these states obtained from ALEPH, DELPHI [21], and OPAL [22] are given in table 9. It can be seen that the predictions for the level spacings are well born out. DELPHI and OPAL have observed the $B_{s}^{* *}$ 
TABLE 8. Quantum numbers of excited (nonstrange) $B$ mesons, using orbital angular momentum. the total anguiar momentum of the light degrees of freedom. and total angular momentum and parity to describe the states.

\begin{tabular}{||c|c|c|c|c||}
\hline$L$ & $j$ & $J^{P}$ & State & $\begin{array}{c}\text { Dominant Decay } \\
\text { Mode }\end{array}$ \\
\hline 0 & $\frac{1}{2}$ & $0^{-}$ & $B$ & \\
0 & $\frac{1}{2}$ & $1^{-}$ & $B^{*}$ & $B \gamma$ \\
1 & $\frac{1}{2}$ & $0^{+}$ & $B_{0}^{*}$ & $B \pi($ S-Wave) \\
1 & $\frac{1}{2}$ & $1^{+}$ & $B_{1}^{*}$ & $B^{*} \pi$ (S-Wave) \\
1 & $\frac{3}{2}$ & $1^{+}$ & $B_{1}$ & $B^{*} \pi$ (D-Wave) \\
1 & $\frac{3}{2}$ & $2^{+}$ & $B_{2}^{*}$ & $B^{*} \pi, B \pi$ (D-Wave) \\
\hline
\end{tabular}

TABLE 9. Masses of the $B^{* *}$ states

\begin{tabular}{|c|c|}
\hline experiment & $\begin{array}{c}\text { Mass } \\
\mathrm{MeV} / \mathrm{c}^{2}\end{array}$ \\
\hline$B_{u, d}^{* *}:$ & \\
ALEPH(91-94) & $5734 \pm 4 \pm 10$ \\
DELP.HI(91-94) & $5734 \pm 5 \pm 17$ \\
OPAL(91-94) & $5712 \pm 11$ (stat) \\
LEP Average & $5727 \pm 6$ \\
$B_{: * *}^{* *}$ & \\
DELPHI $\left(B_{a 1}\right)$ & $5888 \pm 4 \pm 8$ \\
DELPHI $\left(B_{: 2}^{*}\right)$ & $5914 \pm 4 \pm 8$ \\
OPAL & $5884 \pm 15$ \\
\hline
\end{tabular}

decaying into $B^{(*)} K$. The DELPHI result is shown in figure 17 . The two states observed by DELPHI are identified as the $B_{s 1}$ and the $B_{s 2}^{*}$.

DELPHI [23] has also observed two resonances in the state $B^{(*)} \pi^{+} \pi^{-}$. The data are shown in figure 18 . The bump at $Q=301 \mathrm{MeV} / \mathrm{c}^{2}$ is interpreted as evidence for a radial excitation of the $B$ at a mass value of about $5860 \mathrm{MeV} / \mathrm{c}^{2}$. The predicted spectrum is shown in figure 19 and supports this identification.

\section{G The $B_{c}$ Meson}

Finally, we discuss a particle that has not yet been observed, the $B_{c}^{+}$meson. This particle is especially interesting because it can be treated by means of nonrelativistic potential models. Such a treatement [24] gives a prediction for the mass value of the lowest mass states of this family:

$$
M_{B_{c}}=6258 \pm 20 \mathrm{MeV} / \mathrm{c}^{2} \quad M_{B_{e}^{*}}-M_{B_{e}}=73 \mathrm{MeV} / \mathrm{c}^{2}
$$




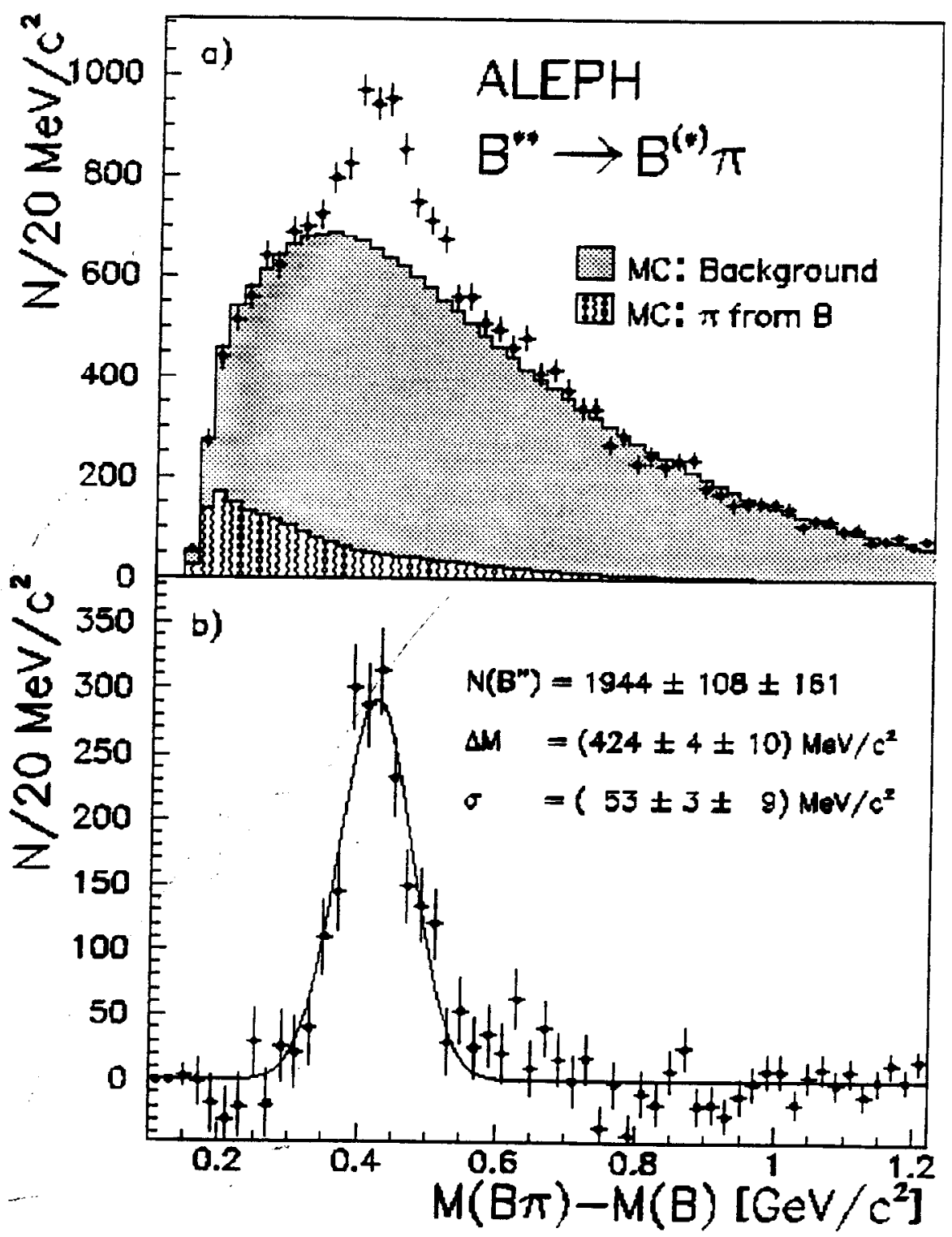

FIGURE 16. a) The $(B \pi)-B$ mass difference distribution from 1991 through 1994 data from ALEPH. The background estimated from the Monte Carlo simulation is shown by the hatched area. b) The background subtracted signal for the decay $B^{* *} \rightarrow B^{(*)} \pi^{ \pm}$fitted with a Gaussian(curve). 


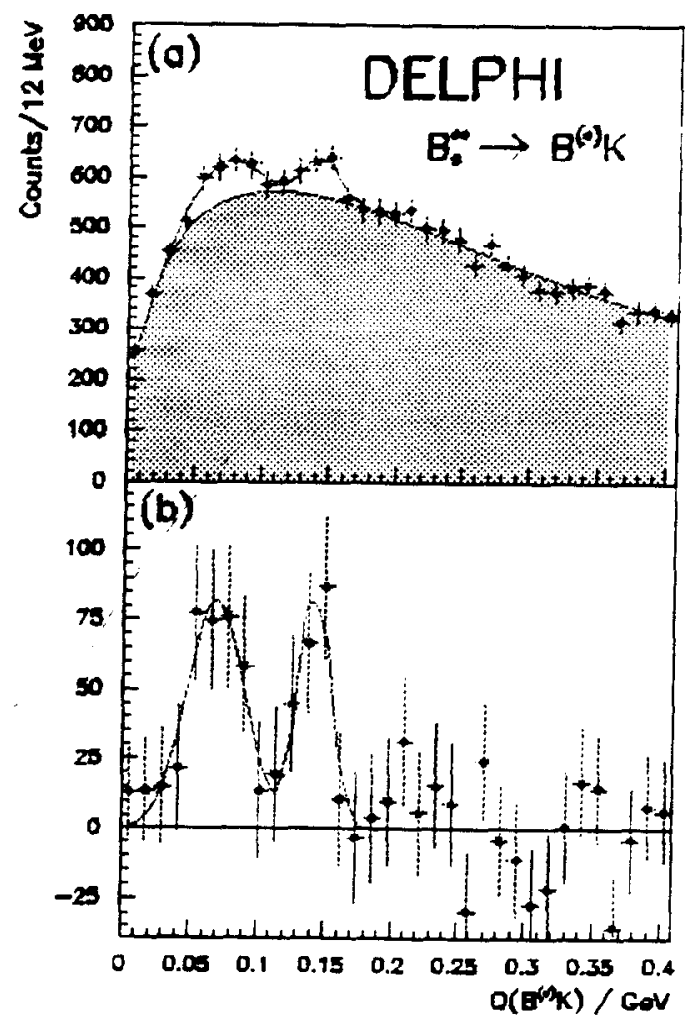

FIGURE 17. Invariant mass difference distribution for $B_{s}^{* *} \rightarrow B^{(*)} K$ from DELPHI. a) Inclusive distribution with estimated background from Monte Carlo showed as shaded area: and b) Background subtracted distribution. 


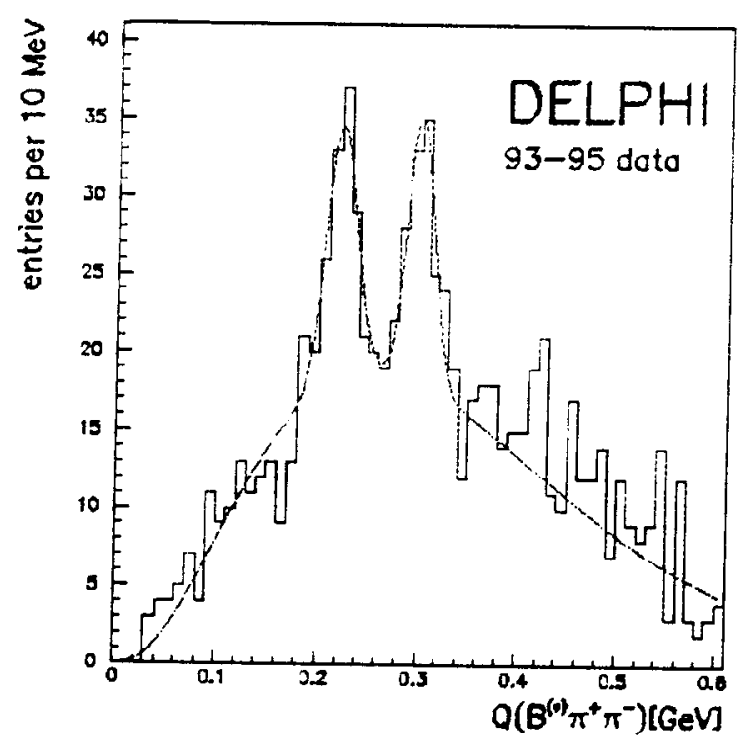

FIGURE 18. Observation of resonances in the $B^{(*)} \pi^{+} \pi^{-}$mass distribution from DELPHI. The quantity plotted on the lower axis, $Q\left(B^{(*)} \pi^{+} \pi^{-}\right)$is $M\left(B^{(*)} \pi^{+} \pi^{-}\right)-M\left(B^{(*)}\right)-2 M(\pi)$.

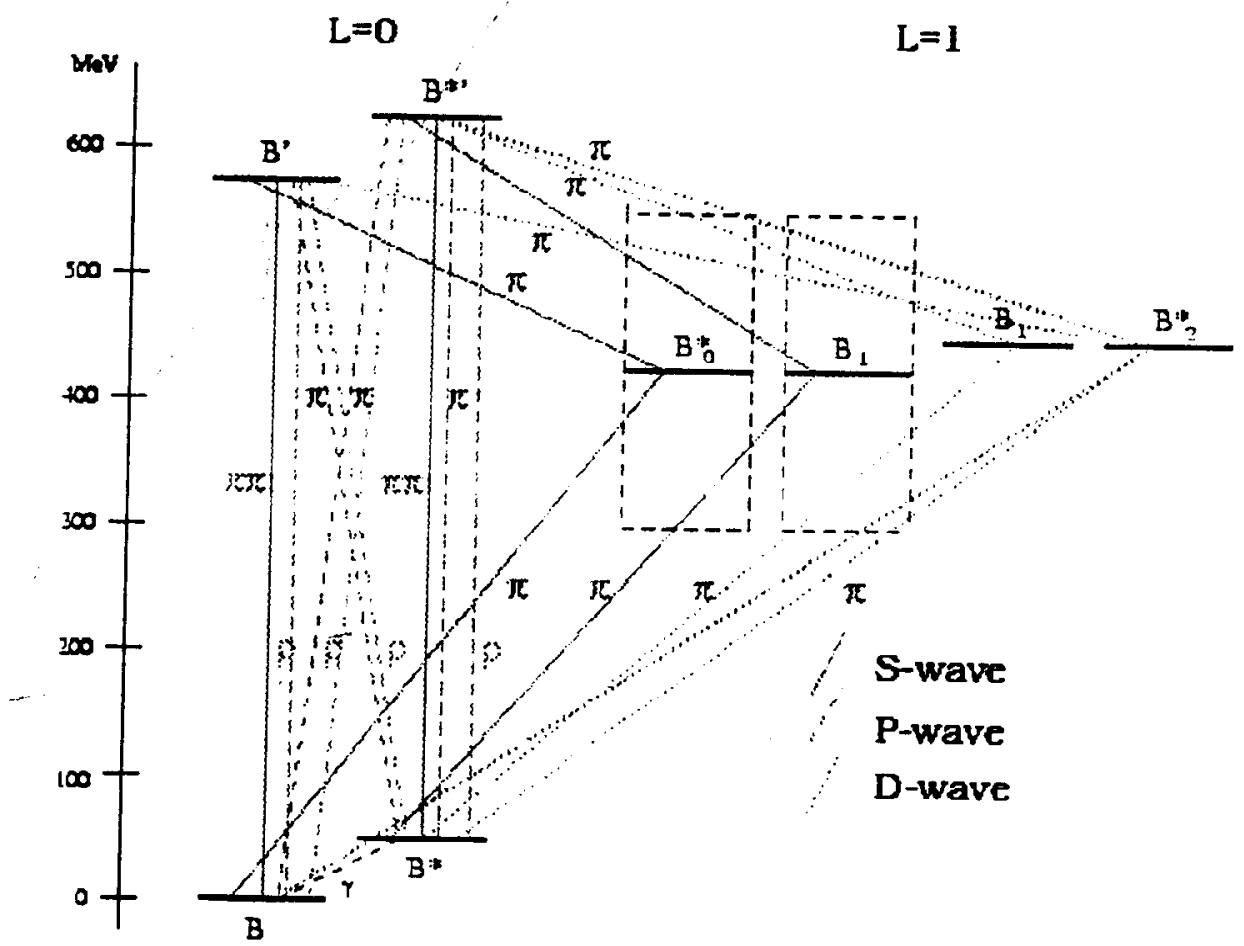

FIGURE 19. Spectrum of excited $B$ mesons showing the radial excitations $B^{\prime}$ and $B^{*^{\prime}}$ decaying to ground state $B$ and $B^{*}$ mesons through the sequential emission of pions. 


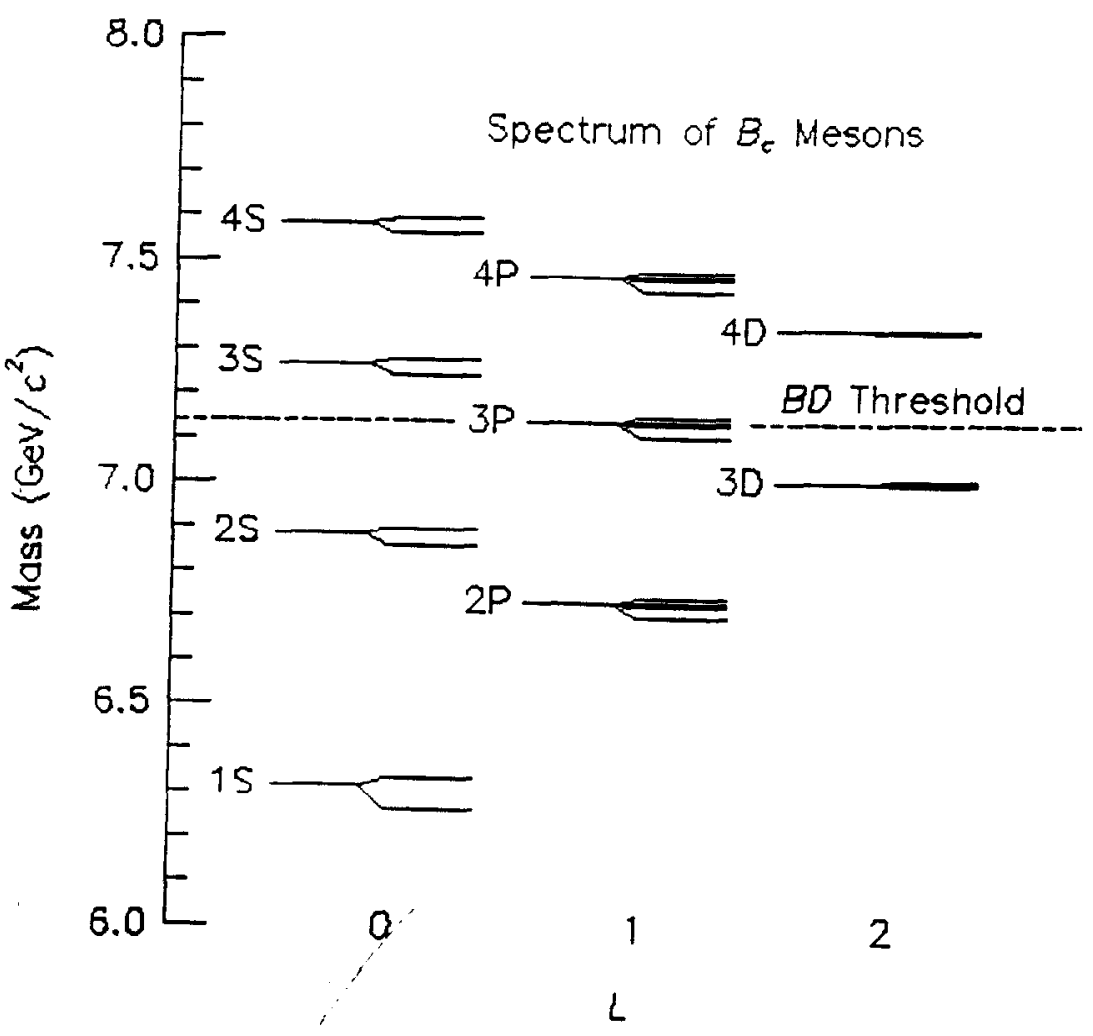

FIGURE 20. Spectrum of $c \bar{b}$ states.

The models predict a spectrum of excited states many of which are below $B D$ threshold and thus decay mainly through radiative transitions. The spectrum of these states is shown in figure 20 .

These states are expected to decay into states containing $\psi$ mesons and this may provide a convenient set of decay modes in which to search for these particles. Predictions of branching fractions for promising decay modes in which to observe these states are given in table 10 .

TABLE 10. Promising

Decay Modes for the Ob-

servation of $B_{c}$ States

\begin{tabular}{|c|c|}
\hline \hline Mode & Br \\
\hline$B_{c} \rightarrow \psi \pi^{\ddagger}$ & $4.0 \times 10^{-3}$ \\
$B_{c} \rightarrow D_{s} \psi$ & $5.0 \times 10^{-2}$ \\
$B_{c} \rightarrow \psi l \nu$ & $10 \%$ \\
\hline \hline
\end{tabular}




\section{H Concluding Remarks on Excited Charm and Bottom Mesons}

The spectroscopy of heavy quarks, while it may be considered to be a mature field which is reasonably weil described by theoretical models, nevertheless still presents subtle and exciting challenges both to experimentalists and to theorists.

The excited states of $D$ and $B$ mesons provide important insights into the properties of hadrons containing heavy quarks through the following relations, which are expected from HQS:

$$
\begin{aligned}
M_{D^{* *}}-M_{D} & \sim M_{B^{* *}}-M_{B} \\
M_{D^{*}}-M_{D} & \sim \frac{M_{b}}{M_{c}} \times\left(M_{B}-M_{B}\right)
\end{aligned}
$$

There are many more measurements one can make including detailed studies of other decay modes and a comparison of their rates relative to the ones already observed and to the predictions of HQS.

Below, it will be seen that an understanding of the hadronic structure of charmed and bottom mesons is necessary to interpret semileptonic (and nonleptonic) decays and to extract Standard Model Parameters, especially CKM elements, from them. HQS will be seen to greatly simplify the problem of disentagling the weak interaction properties of the heavy quarks from the influence of the strong interactions with the accompanying light quarks.

\section{LIFETIMES OF CHARMED AND BOTTOM PARTICLES}

The lifetimes of the charm and bottom particles provide a good 'overview' of several important features of heavy quark decays. This may seem surprising since they involve a summation over all of the many decay channels which are allowed. Nevertheless, many important insights have been gained by a study of lifetimes.

When charmed mesons were first observed, it was generally expected that their decay rate would be roughly that of a free charm quark. This gave rise to the 'spectator model' - the quark decayed via a $W$ emission and the $W$ had no direct interaction with the original light quark which was named the spectator quark because it did not change during the decay process. A typical spectator decay is shown in figure 21 .

In this picture, all charmed particles would have similar lifetimes. As soon as lifetime measurements became available, it was clear that the spectator model was not correct. The $D^{+}$lifetime was more than twice the $D^{\circ}$ lifetime. Moreover, the $\Lambda_{c}^{+}$lifetime was much shorter than the $D^{\circ}$ lifetime. Table 11 shows the world-average measurements of the charmed meson lifetimes [25]. 


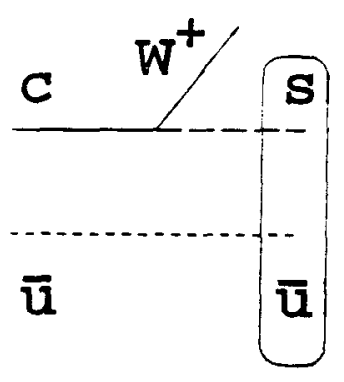

FIGURE 21. Spectator decay of the $D^{\circ}$ meson.

TABLE 11. Lifetimes of charmed mesons

\begin{tabular}{|c|c|}
\hline Meson & $\begin{array}{c}\text { lifetime } \\
\text { ps }\end{array}$ \\
\hline$D^{+}$ & $1.057 \pm 0.015$ \\
$D^{\circ}$ & $0.415 \pm 0.004$ \\
$D_{s}^{+}$ & $0.467 \pm 0.017$ \\
\hline
\end{tabular}

Many attempts were made to explain the pattern of lifetimes of the charmed mesons and baryons. The solution to the problem required taking into account additional quark level processes which can contribute to the weak decays and the quantum effects associated with them. There are six such processes whose diagrams are shown in figure 22.

These diagrams have different dependences on the heavy quark mass. The spectator diagram goes like the mass to the fifth power, $M_{Q}^{5}$, which is required by dimensional analysis and which is analogous to muon decay. The other processes all require the two valence quarks to be within the range of the virtual $W$ and therefore depend on the wave function squared of the initial state heavy meson or baryon at the origin. Since the wave function squared has the dimension

$$
-|\psi(0)|^{2} \propto \frac{1}{l e n g t h^{3}} \propto M_{Q}^{3}
$$

the dependence of all these diagrams on the heavy quark mass goes like $M_{Q}^{2}{ }^{5}$ Therefore, as the quark mass gets heavy, the relative importance of the nonspectator diagrams to the spectator diagram should decrease.

In fact, there are two diagrams which are classified as 'spectator diagrams' in figure 22:

- One is just the simple diagram shown above with the $W$ decaying into quarks which form hadrons without any communication with other fi-

5) Various QCD effects like gluon enission which can relieve the helicity suppression or color suppression can change this dependence to $M_{Q}^{2}$. 


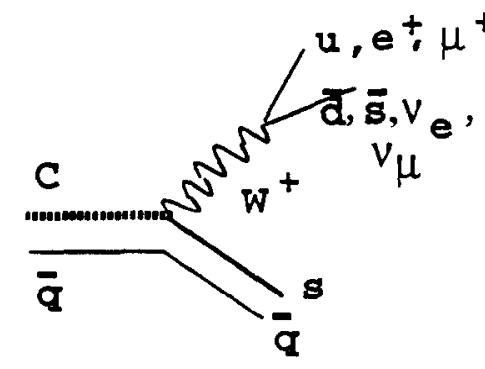

a) simple spectator
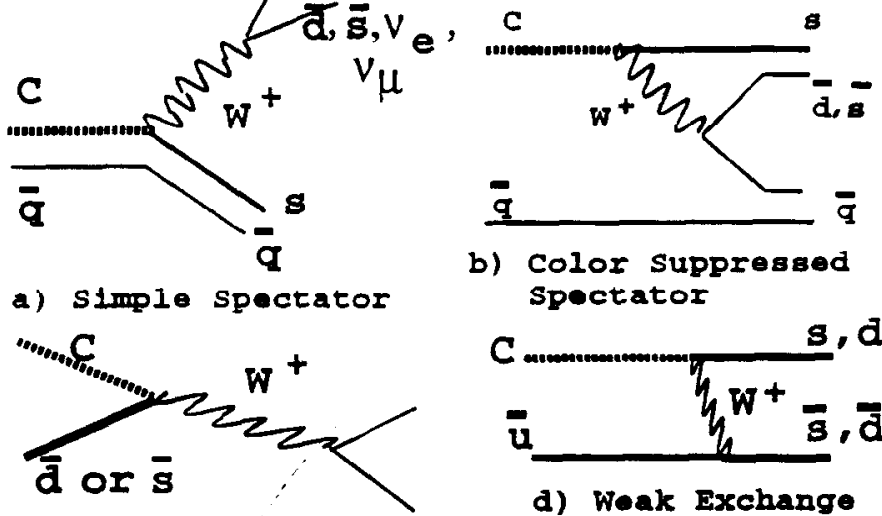

b) color suppressed

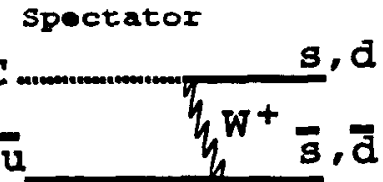

c) Weak Annihilation

d) Weak Exchange
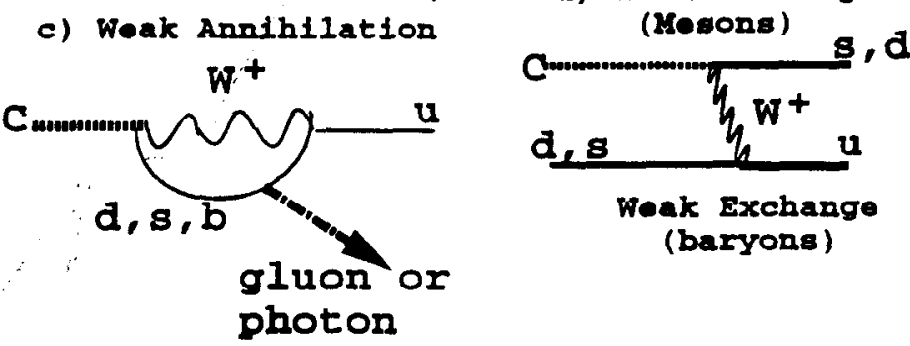

-) Penguia (Loop)

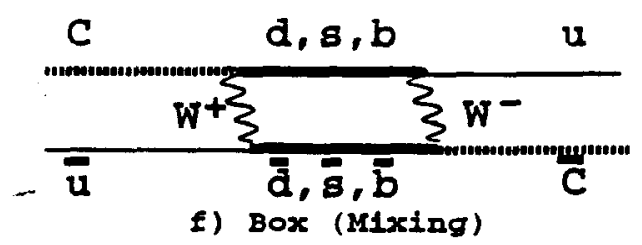

FIGURE 22. Quark level diagrams for weak decays 
nal state quarks. This is called the 'external W emission' or 'external spectator' diagram.

- In the second diagram, the quarks from the $\mathrm{W}$ decay combine with the spectator quark and with the final state quark that coupled directly to the W. This is the so-called 'internal W emission' or 'internal spectator' diagram.

For final states where both diagrams are possible, if one of the light antiquarks in the $W$ decay is the same as the spectator antiquark, then interference effects are possible. Another important consideration is that the quarks from the 'internal W emission' must match color of the other two quarks in the final state. This restricts the number of possibilities and reduces the overall contribution to decay amplitude by a factor of 3 . Because of this, the 'internal emission' diagram is often referred to as the 'color-suppressed' diagram. However, gluon radiation in the initial or final state can disrupt the color suppression so the degree of suppression is not well-predicted and the two amplitudes could turn out to be 'comparable'.

\section{A Charmed Meson Lifetimes}

What is responsible for the lifetime differences among the charmed mesons? In particular, why is the $D^{+}$lifetime so much longer than the $D^{\circ}$ lifetime? It has to be the result of some kind of destructive interference effect between the amplitudes described above. In meson decay, the W-exchange and annihilation amplitudes are suppressed by helicity and color considerations and are believed to be too small to be the cause of the observed differences. It is natural to look for the answer in the two spectator decay diagrams.

A key difference between $D^{+}$and $D^{\circ}$ decays is that the Cabibbo-favored $D^{+}$decays can experience light quark interference but the (CF) $D^{\circ}$ decays cannot [26]. The situation is shown in figure 23 for two body decays. The two diagrams for the $D^{+}$can interfere destructively reducing the decay rate and increasing the lifetime. In the figure, the decay final states are divided into three types:

- TYPE I: Decays which can only proceed through external W emission with an amplitude $a_{1}$;

- TYPE II: Decays which can only proceed through internal W emission with an amplitude $a_{2}$; and

- TYPE III: Decays which can proceed through both internal and external $W$ emission and will depend on the magnitude $a_{1}$ and $a_{2}$ and their relative sign which determines whether the interference is constructive or destructive. 
Only the $D^{+1}$ s have Cabibbo-favored TYPE III decays. This results in a suppression of some Cabibbo-favored two body decay modes. Since a large fraction of all charm decays are two body or quasi-two body, this could result in an overall increase in the $D^{+}$lifetime relative to the $D^{\circ}$. However, it is not very satisfying to extrapolate from an argument based on two body decays to the full decay rate.

It is also possible to make a simlar 'inclusive' argument based on quark level dynamics alone. For the Cabibbo-favored decays, we have $D^{\circ} \rightarrow$ sud $\bar{u}$ and $D^{+} \rightarrow$ sud $\bar{d}$. Simlarly, we have $B^{\circ} \rightarrow c d \bar{u} \bar{d}$ and $B^{+} \rightarrow c d \bar{u} \bar{u}$. In each case, the internal and external diagrams can interfere. Let $c_{1}$ be the amplitude for external $W$ emission and $c_{2}$ be the amplitude for internal emission. Then for the $D^{\circ}$ (and $B^{\circ}$ ), the (nonleptonic) decay width, $\Gamma_{N L}$, is given by

$$
\Gamma_{N L}\left(D^{\circ} \text { or } B^{\circ}\right) \propto 3\left(c_{1}^{2}+c_{2}^{2}+2 \xi^{\prime} c_{1} c_{2}\right) G_{F}^{2} M_{c \text { or b }}^{5} / 192 \pi^{3}
$$

where $\xi^{\prime}$ is the degree of color coherence between the diagrams. However, for the $D^{+}\left(B^{+}\right)$, the $\bar{d}(\bar{u})$ spectator antiquark and antiquark from the $W$ decay can also interfere adding extra terms to the decay rate. The expression for the decay rate is:

$$
\begin{aligned}
\Gamma_{N L}\left(D^{+} \text {or } B^{+}\right) \propto & 3 G_{F}^{2} M_{c \text { or } b}^{2}\left[\left(c_{1}^{2}+c_{2}^{2}+2 \xi^{\prime} c_{1} c_{2}\right) M_{c \text { or } b}^{3} / 192 \pi^{3}\right. \\
& \left.+\left(2 c_{1} c_{2}+\xi^{\prime}\left(c_{1}^{2}+c_{2}^{2}\right)\right)|\psi(0)|^{2} / \pi\right]
\end{aligned}
$$

The extra term is proportional to $M_{Q}^{2}$ and the value of the wave function at the origin and.which is destructive, at least in the case of charm. This contribution, called 'Pauli interference', can explain the lifetime differences.

Since some two body decays proceed only via external emission, some by only internal emission, and some through both, it is possible to determine the parameters $a_{1}$ and $a_{2}$. The study of two body charm decays gives the result [27] that:

$$
\frac{a_{2}}{a_{1}}=-(0.4 \pm 0.1)
$$

These are related to $c_{1}$ and $c_{2}$ as follows:

$$
\begin{aligned}
& a_{1}=c_{1}+\xi c_{2} \\
& a_{2}=c_{2}+\xi c_{1}
\end{aligned}
$$

The quantity $\xi$ is not necessarily the same as $\xi^{\prime}$ in the inclusive treatment. The amplitudes do indeed interfere destructively and are 'comparable'. Models based on light quark interference in $D^{+}$decays can explain the lower $D^{+}$decay rate (longer lifetime) and this is considered to be the correct explanation for this puzzle.

For $B$-decays, a recent analysis [28] of the measured two body decays gives 


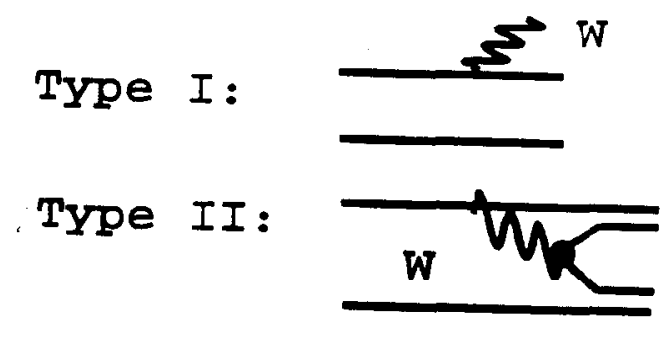

Type III: Both $\rightarrow$ interference possible

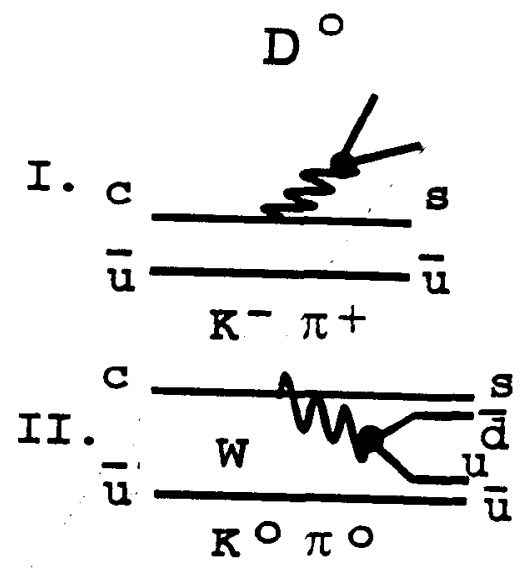
$\mathrm{D}^{+}$

III.
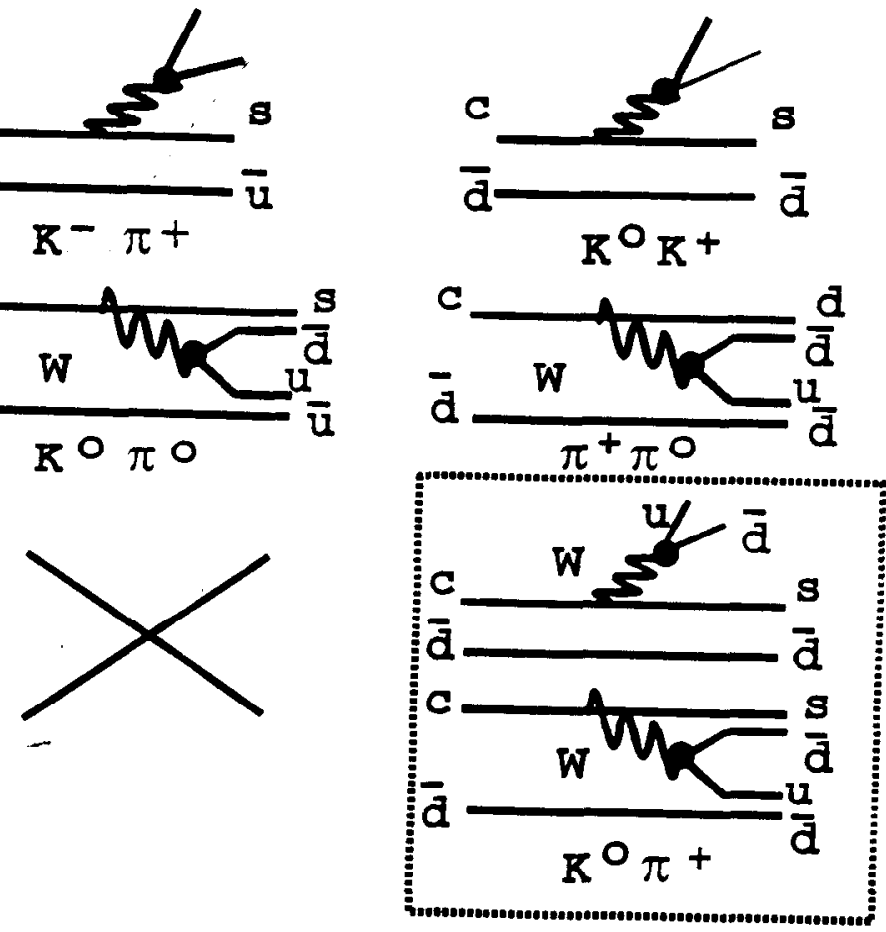

FIGURE 23. Comparison of the Cabibbo-favored spectator diagrams contributing to the decay of the $D^{\circ}$ and $D^{+}$mesons. 


$$
\frac{a_{2}}{a_{1}}=+(0.26 \pm 0.08 \pm 0.06)
$$

The positive sign suggests that the interference may be constructive which is very surprising. If that is true, the $B^{+}$might have a shorter lifetime than the $B^{\circ}$. More accurate measurements are needed to verify this unexpected prediction.

\section{B Charmed Baryon Lifetimes}

There are significant differences between meson decay and baryon decay. The meson decays are constrained by color considerations and by helicity suppression. All three colors are present in baryons as are three spins so neither color suppression nor helicity suppression play a role whereas they are important factors in meson decays. Because the nonspectator diagrams are unsuppressed, their effect is expected to show up more dramatically in charmed baryon decays than in charmed meson decays.

The nonleptonic decays of the four weakly decaying singly charmed baryons may be written, in the spirit of the inclusive analysis described above, as:

$$
\begin{gathered}
\Gamma_{N L}\left(\Lambda_{c}^{+}\right)=\Gamma^{\operatorname{dec}}\left(\Lambda_{c}^{+}\right)+\Gamma^{\mathrm{exch}}\left(\Lambda_{c}^{+}\right)_{d}+\Gamma_{-}^{\text {int }}\left(\Lambda_{c}^{+}\right)_{u} \\
\Gamma_{N L}\left(\Xi_{c}^{+}\right)=\Gamma^{\operatorname{dec}}\left(\Xi_{c}^{+}\right)+\Gamma_{-}^{\text {int }}\left(\Xi_{c}^{+}\right)_{u}+\Gamma_{+}^{\text {int }}\left(\Xi_{c}^{+}\right)_{d} \\
\Gamma_{N L}\left(\Xi_{c}^{o}\right)=\Gamma^{\operatorname{dec}}\left(\Xi_{c}^{o}\right)+\Gamma^{\text {exch }}\left(\Xi_{c}^{o}\right)_{d}+\Gamma_{+}^{\text {int }}\left(\Xi_{c}^{o}\right)_{s} \\
\Gamma_{N L}\left(\Omega_{c}^{o}\right)=\Gamma^{\operatorname{dec}}\left(\Omega_{c}^{o}\right)+\Gamma_{+}^{i n t}\left(\Omega_{c}^{o}\right)_{o}
\end{gathered}
$$

Here, $\Gamma^{\text {dec }}$ is just the spectator decay. The 'exchange' contribution and the 'constructive' light quark interference term labelled $\Gamma_{+}^{\text {int }}$ add to the width and decrease the lifetime and the 'destructive' light quark interference term $\Gamma_{-}^{\text {int }}$ increases the lifetime. With these expressions, one can deduce the 'lifetime hierarchy' of the charmed baryons. For example, Guberina, Ruckl, and Trampetic [29] estimate

$$
\Gamma^{\text {exch }}: \Gamma_{+}^{\text {int }}: \Gamma_{-}^{\text {int }} \simeq 1: 0.5:-0.3
$$

and, in a more detailed calculation, predict

$$
\tau\left(\Xi_{c}^{\circ}\right): \tau\left(\Lambda_{c}^{+}\right): \tau\left(\Xi_{c}^{+}\right) \propto 0.6,1.0,1.6
$$

E687 at Fermilab has measured all four of these lifetimes. The world-average results and E687 results are presented in table 12 . It should be noted that the $\Omega_{c}$ appears to be the shortest lived of the charmed baryons. Also, 


$$
\frac{\tau\left(\Xi_{c}^{+}\right)}{\tau\left(\Lambda_{c}^{+}\right)}=2.15 \pm 0.59 \text { and } \frac{\tau\left(\Lambda_{c}^{+}\right)}{\tau\left(\Xi_{c}^{o}\right)}=1.89_{-0.48}^{+0.35}
$$

Obviousiy, more statistics will help sharpen this comparison but the theory seems to reproduce the trend of the data. In particular, significant contributions from exchange diagrams and interference effects are required to explain the charmed baryon lifetime hierarchy.

TABLE 12. World average and E687 values of the lifetimes of charmed baryons [30].

\begin{tabular}{||c|c|c||}
\hline Baryon & $\begin{array}{c}\text { lifetime (PDG) } \\
\text { ps }\end{array}$ & $\begin{array}{c}\text { lifetime (E687) } \\
\text { ps }\end{array}$ \\
\hline$\Lambda_{c}^{+}$ & $0.206 \pm 0.012$ & $0.215 \pm 0.01 \pm 0.008$ \\
$\Xi_{c}^{+}$ & $0.35_{-0.04}^{+0.07}$ & $0.41_{-0.08}^{+0.11} \pm 0.02$ \\
$\Xi_{c}^{0}$ & $0.098_{-0.015}^{+0.023}$ & $0.101_{-0.017}^{+0.025} \pm 0.005$ \\
$\Omega_{e}^{0}$ & $0.064 \pm 0.020$ & $0.086_{-0.020}^{+0.027} \pm 0.028$ \\
\hline
\end{tabular}

\section{B Meson and Baryon Lifetimes}

The $B$ meson lifetimes have been a topic of intense investigation at $e^{+} e^{-}$ machines and at CDF at the Fermilab Tevatron. From the picture presented above, one expects a further suppression of nonspectator effects of about a factor of 10-20 because of the large mass of the $b$-quark. The lifetimes of the $B$-mesons should all be very similar, with the $B^{+}$being slightly longer lived than the $B^{\circ}$ due to interference. However, the recent analysis of two body $B$-meson decays from CLEO, described above, suggests that the Pauli interference is constructive in $B^{+}$decays. If this surprising result is true, then the $B^{+}$may turn out to be shorter lived than the $B^{\circ}$ ! The baryons are expected to be about $10 \%$ shorter lived than the mesons. The general picture is certainly borne out by the data given in table 13, at least to the extent that the fractional lifetime differences among the various species of $B$-hadrons are certainly much smaller than the differences among the corresponding species of charmed hadrons. More accurate measurements will tell us whether the detailed predictions of the small differences among the $B$-hadron species are borne out.

\section{SEMILEPTONIC AND LEPTONIC DECAYS}

About $20 \%$ of the time, a $D^{\circ,+}$ or a $B^{\circ,+}$ decays semileptonically. Weak semileptonic decays are among the simplest decays to describe theoretically because: 
TABLE 13. Resuits on lifetimes of $B$ mesons and baryons [31].

\begin{tabular}{|c|l|l|l|l|}
\hline \hline & LEP Avg & CDF & SLD & Worid Avg \\
\hline$b$-quark & $1.54 \pm 0.02$ & $1.51 \pm 0.03$ & $1.56 \pm 0.05$ & $1.53 \pm 0.02$ \\
$B^{-}$ & $1.63 \pm 0.06$ & $1.68 \pm 0.07$ & & $1.65 \pm 0.05$ \\
$B^{\circ}$ & $1.52 \pm 0.06$ & $1.58 \pm 0.09$ & & $1.55 \pm 0.05$ \\
$B_{s}$ & $1.60 \pm 0.10$ & $1.36 \pm 0.12$ & & $1.50 \pm 0.08$ \\
$A_{b}$ & $1.21 \pm 0.07$ & $1.32 \pm 0.17$ & & $1.23 \pm 0.06$ \\
$\Xi_{b}$ & $1.39_{-0.28}^{+0.34}$ & & & \\
\hline \hline
\end{tabular}

a)

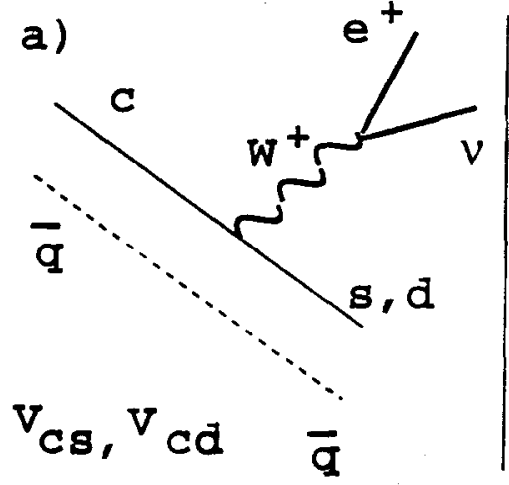

b)

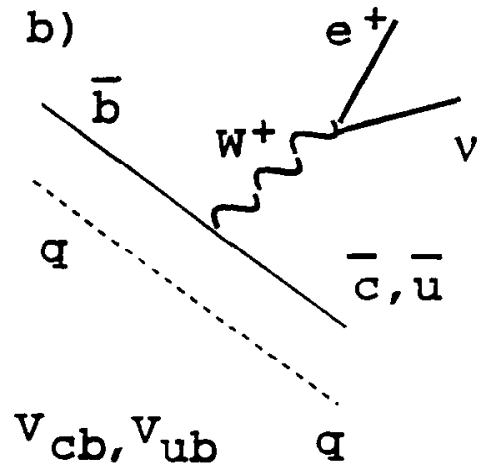

FIGURE 24. Schematic representation on the decay of a) a charmed or b) a bottom meson.

- Only the spectator diagram contributes because the leptons coming from the virtual $W$ do not interact with the remaining quarks at all;

- The leptonic vertex is completely determined by the theory of the electroweak interaction; and

- The strong interaction effects, which come from the requirement that the initial and final quarks be bound into hadrons, can be described by one or more form factors which depend only on $q^{2}$, the mass of the virtual $W$ which is responsible for the decay.

These decays are shown schematically in figure 24. The number of form factors. which describe the hadronic vertex in the decay of a pseudoscalar meson depends on the spin of the outgoing hadron.

The matrix element for a semileptonic decay is given by

$$
\begin{aligned}
A(M \rightarrow \text { mev }) & =\frac{G_{F}}{\sqrt{2}} V_{Q_{q}} L^{\mu} H_{\mu}, \text { where } \\
L^{\mu} & =\bar{u}_{e} \gamma^{\mu}\left(1-\gamma_{5}\right) v_{\nu}, \text { and } \\
H^{\mu} & =\left\langle m\left|J_{h a d}^{\mu}(0)\right| M>.\right.
\end{aligned}
$$

The semileptonic decay rate is proportional to the square of a CKM matrix element. It is possible to extract CKM elements from measurements of the 
semileptonic width and this is, in fact, the preferred method. However, the value of a CKM matrix element always appears in a product with the hadronic form factor. To obtain the value of the CKM parameter, we must have a model which predicts the form factor and allows us to divide out its effect. This represents a challenge for nonperturbative QCD. Today, extraction of CKM parameters from data is often limited by the uncertainties in the theoretical estimate of the form factors rather than by the statistical or systematic errors of the measurement of the the semileptonic width.

In the charm system, the CKM matrix elements are given by the Cabibbo angle which can be determined from $K$ meson decay. This permits us to use charm semileptonic decays to extract the hadronic matrix elements without relying on models. We then have the following game plan:

- Use charm semileptonic decays where we 'know' $V_{c s}$ and $V_{c d}$ to isolate the properties of the hadronic vertex;

- Compare these results to the predictions of various quark models and lattice gauge calculations;

- Establish confidence in at least some of these approaches; and

- Armed with the experience, use theoretical calculations to extract $V_{c b}$ and $V_{u b}$ from measurements of $B$ semileptonic decay.

Since the quantity $q^{2}$ plays an important role in the study of semileptonic decays, it is worth understanding the range which can be assumed by this variable and the physical configuration corresponding to its various values. Figure 25 shows the minimum $q^{2}$ configuration and the maximum $q^{2}$ configuration. In the minimum $q^{2}$ configuration ( $q^{2} \sim 0$ if the lepton is an electron), the recoiling final state meson is moving fast and is relativistic which causes problems for theory. In the maximum $q^{2}$ configuration, the final state hadron is at rest in the rest frame of the parent meson and is nonrelativistic so theory is happy. Unfortunately, it is often difficult for experiments to measure out to high $q^{2}$. The maximum value of $q^{2}$ is given by

$$
q_{\max }^{2}=(M-m)^{2}
$$

where $M$ is the mass of the parent hadron and $m$ is the mass of the daughter hadron. Table 14 gives the value of this important quantity for typical decays.

\section{A Pseudoscalar to pseudoscalar decay}

The formula for the semileptonic decay rate of pseudoscalar meson to a lighter pseudoscalar meson is [32]:

$$
\frac{d^{2} \Gamma}{d q^{2}}=\frac{G_{F}^{2}\left|V_{Q_{q}}\right|^{2}|h|^{3}}{24 \pi^{3}}\left[\left|f_{+}\left(q^{2}\right)\right|^{2}+m_{l}^{2}\left|f_{-}\left(q^{2}\right)\right|^{2} \ldots\right]
$$




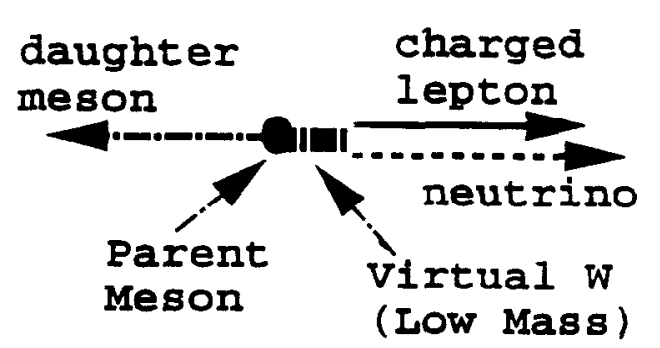

(a)

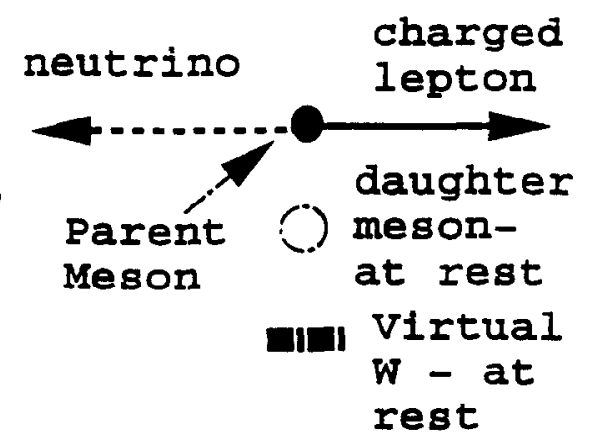

(b)

FIGURE 25. Final state particle configurations for a) minimum $q^{2}$ and b) maximum $q^{2}$.

TABLE 14. Maximum value of $q^{2}$ for various semileptonic decays

\begin{tabular}{|l|c|}
\hline & \\
\hline \hline Decay & $q_{\max }^{2}\left(\mathrm{GeV} / \mathrm{c}^{2}\right)$ \\
\hline$D^{\circ} \rightarrow K^{-l^{+} \nu}$ & 1.88 \\
$D^{\circ} \rightarrow \pi^{-} l^{+} \nu$ & 3.00 \\
$D^{+} \rightarrow K^{* \circ} l^{+} \nu$ & 0.95 \\
$B^{+} \rightarrow D^{\circ} l^{+} \nu$ & 11.65 \\
$B^{\circ} \rightarrow D^{*+} l^{+} \nu$ & 10.60 \\
\hline \hline
\end{tabular}

where

$$
h=\frac{M}{2}\left[\left(1-\frac{m^{2}}{M^{2}}-y\right)^{2}-4 \frac{m^{2}}{M^{2}} y\right]^{1 / 2}
$$

and $y=q^{2} / M^{2}, M$ is the mass of the parent meson, and $m$ is the mass of the daughter meson. $h$ is the magnitude of the momentum of the daughter meson $m$ in the rest frame of the parent meson $M .|h|^{3}$ is large when $q^{2}$ is small and small when $q^{2}$ is large. The $f_{-}$form factor contribution vanishes for zero mass leptons and is negligible for electrons but may begin to be felt for muons.

The form factor is usually approximated by a function like

$$
f_{+}\left(q^{2}\right)=\frac{f_{+}(0)}{\left(1-q^{2} / M_{p}^{2}\right)}
$$

or something like

$$
f_{+}\left(q^{2}\right)=f_{+}(0) \times \exp ^{-\alpha q^{2}}
$$

The pole mass, $M_{p}$, is expected, by a duality argument, to be the lowest mass charmed vector or axial vector meson whose quantum numbers correspond to those of the hadron vertex. For example, figure 26 shows why the $D_{s}^{*}$, whose mass is $2112 \mathrm{MeV} / \mathrm{c}^{2}$, is expected to be the dominant pole contributing to the 


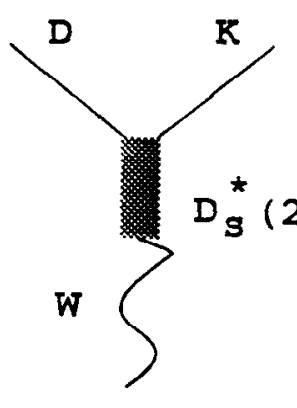

(a)

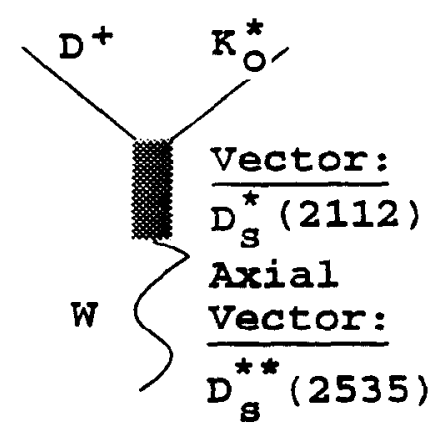

(b)

FIGURE 26. Illustration of pole contribution to hadronic vertex

TABLE 15. $K l \nu$ Form factor measurements [35]

\begin{tabular}{|c|c|c|c|}
\hline Exp. & mode & $\overline{M_{\text {pole }}}$ & $\overline{f_{+}(0)}$ \\
\hline E691 & $K^{-} e^{+} \nu_{e}$ & $2.1_{-0.2}^{+0.4} \pm 0.2$ & $0.79 \pm 0.05 \pm 0.06$ \\
\hline CLEO(91) & $K^{-} e^{+} \nu_{e}$ & $2.1_{-0.2-0.2}^{+0.4+0.3}$ & $0.81 \pm 0.03 \pm 0.06$ \\
\hline CLEO(93) & $K-l+\nu_{l}$ & $2.0 \pm 0.12 \pm 0.18$ & $0.77 \pm 0.01 \pm 0.04$ \\
\hline MKIII & $K^{-} e^{+} \nu_{e}$ & $1.8_{-0.2-0.2}^{+0.5+0.3}$ & $\left|V_{c s}\right|(0.72 \pm 0.05 \pm 0.04)$ \\
\hline E687 & $K^{-} \mu^{+} \nu_{\mu}$ (tág) & $1.97_{-0.22-0.06}^{+0.43+0.07}$ & $0.71 \pm 0.05 \pm 0.03$ \\
\hline E687 & $K^{-} \mu^{+} \nu_{\mu}($ inc $)$ & $1.87_{-0.08-0.06}^{+0.11+0.07}$ & $0.71 \pm 0.03 \pm 0.02$ \\
\hline
\end{tabular}

decay $D^{\circ} \rightarrow K_{-}^{-} l^{+} \nu$. Note that the pole term increases as $q^{2}$ increases but the $|h|^{3}$ term falls off rapidly and wins out causing the $q^{2}$ distribution to be peaked at low $q^{2}$.

The semileptonic decay width is proportional to $\left|V_{Q_{q}}\right|^{2} \times\left|f_{+}(0)\right|^{2}$. To derive the CKM matrix element from the measured width one must have some model for the form factor. The form factor reflects the probability for the quark from the weak decay and the spectator quark to 'fit into' the wave function of the final state hadron.

$$
1 \quad D^{\circ} \rightarrow K^{-} l^{+} \nu
$$

Figure 27 shows the $q^{2}$ distribution obtained by CLEO [33] and by E687 [34]. Table 15 lists recent results for the form factor values at $q^{2}=0$ and the pole masses obtained from fitting the $q^{2}$ distributions. The most recent measurements tend to favor values of the pole mass which are lower than $D_{j}^{*}$.

The measured value of $f_{+}(0)$ agrees well with a variety of quark model and lattice gauge calculations whose predictions range from 0.6 to 0.8 . 

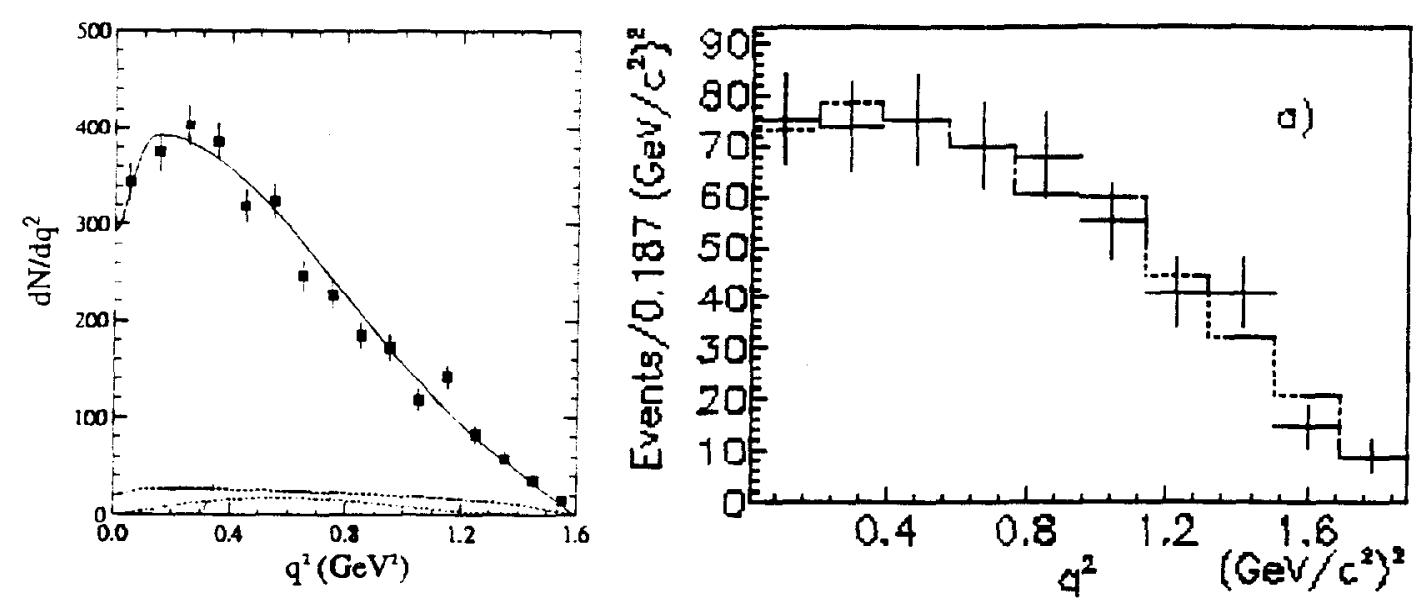

FIGURE 27. $q^{2}$ Dependence for the decay $D^{\circ} \rightarrow K^{-l^{+}} \nu$ from left side: CLEO and right side: $\mathrm{E} 687$.

$$
\text { i } \quad D^{\circ} \rightarrow \pi^{-} \mu^{+} \nu
$$

Figure 28 shows how different models of the pole behavior affect the predicted $q^{2}$ dependence. In the Cabibbo favored semileptonic decays, the $q^{2}$ range is limited to less than 2.0 and the predicted $q^{2}$ dependence differs only slightly for pole masses within the expected range. The Cabibbo suppressed decay has a larger allowed range of $q^{2}$ and, at large $q^{2}$, there is a big difference in the value of the form factor for various choices of the pole mass.

Measurements of $D^{\circ} \rightarrow \pi^{-} \mu^{+} \nu$ are finally becoming available. Figure 29 shows the signals for these decays obtained from E687 [36]. The signal is

qsq vs. ffl and ff2 (form_factor_comp.xx)

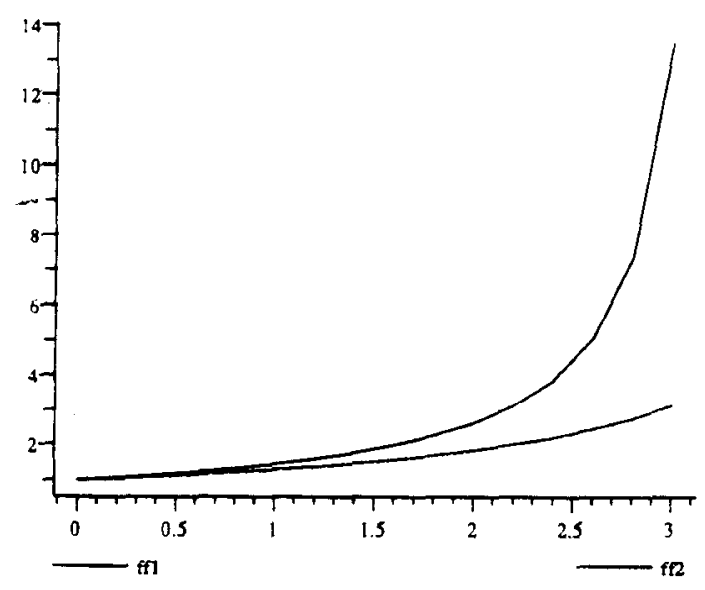

FIGURE 28. Behavior of form factors for two values of the pole mass: $1.8 \mathrm{GeV} / \mathrm{c}^{2}$ (solid line) and $2.1 \mathrm{GeV} / \mathrm{c}^{2}$ (dotted line). 
TABLE 16. $V_{c b}$ Measurements from $B^{\circ} \rightarrow D^{+} e^{-} \bar{\nu}$

\begin{tabular}{|c|l|l|l|}
\hline \hline Model & $f_{+}(0)$ & $V_{c b} f_{+}(0) \times 10^{3}$ & $V_{c b} f_{+}(0) \times 10^{3}$ \\
\hline WB & 0.69 & $25.7 \pm 1.4 \pm 1.7$ & $37.3 \pm 2.0 \pm 2.5$ \\
KS & 0.70 & $25.7 \pm 1.4 \pm 1.7$ & $36.7 \pm 2.0 \pm 2.5$ \\
Demchuk & 0.68 & $24.8 \pm 1.4 \pm 1.6$ & $36.4 \pm 1.6 \pm 2.4$ \\
Average & & & $36.9 \pm 3.7 \pm 0.5$ \\
& & & \\
\hline
\end{tabular}

isolated by finding $\pi^{-} \mu^{+}$or $\pi^{-} e^{+}$vertices downstream of the primary vertex, then using the direction of the parent $D^{\circ}$ obtained from the line connecting the primary and secondary vertex to balance $p_{t}$ about the $D^{\circ}$ flight direction. This determines the kinematics of the missing neutrino (up to a two-fold ambiguity). The signal is then confirmed by finding a pion from the primary vertex which forms a good $D^{*+}$ with the $D^{\circ}$ semileptonic candidate. The signal appears as a peak in the mass difference distribution between the mass of the candidate semileptonic decay and the mass of the candidate combined with a correctly signed pion from the primary vertex which forms the $D^{*}$ parent.

A comparison of the branching fractions and form factors of $\pi l \nu$ to $K l \nu$ is given in figure 30 .

$$
3 \quad \bar{B}^{\circ} \rightarrow D^{+} e^{-} \bar{\nu}
$$

Figure 31 shows the mass distribution obtained by the CLEO collaboration [38] for the $D^{+} e^{-} \bar{\nu}$ final state. In this analysis, the beam constraint and missing energy are used to obtain the neutrino momentum vector. We defer presentation of the $q^{2}$ dependence of the form factor until after a discussion below of Heavy Quark Effective Theory. The branching fraction for the decay is

$$
-1.78 \pm 0.20 \pm 0.24 \%
$$

Table 16 gives values obtained for $V_{c b}$ from this measurement [39]. To obtain the partial width, one gets the total width from the measured value of the $B^{\circ}$ lifetime, here taken to be $1.55 \pm 0.05 \mathrm{ps}$.

\section{B Pseudoscalar to vector decay}

The semileptonic decay of a heavy pseudoscalar meson, $M_{p}$, to a lighter vector meson, $M_{v}$, which subsequently decays into a pseudoscalar, $m$, and a pion via the strong interaction depends on four variables shown in figure 32 : 


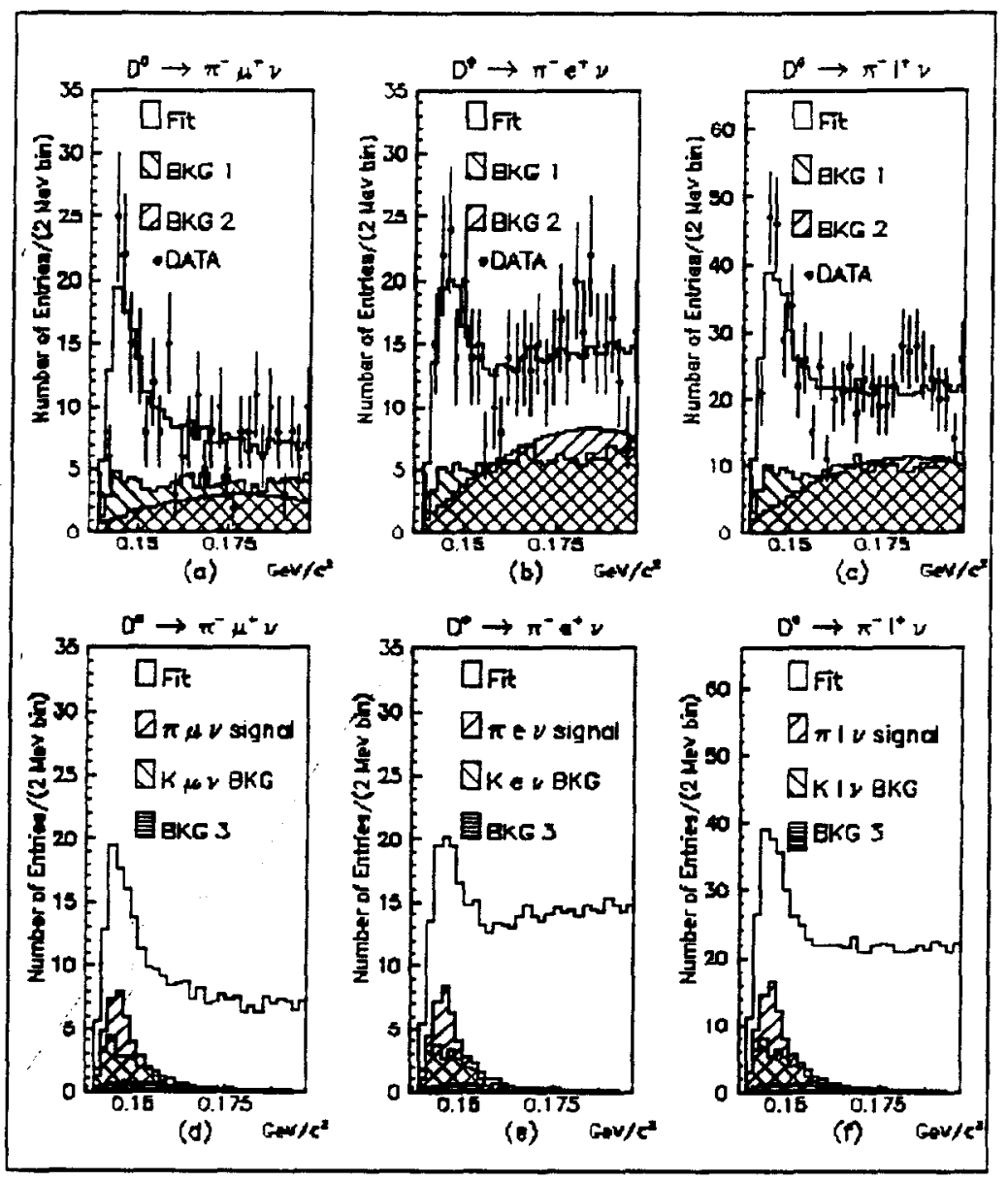

FIGURE 29. Signals for the decay $D^{\circ} \rightarrow \pi^{-} l^{+} \nu$ from E687. a) shows the $D^{*}$ signal for candidate events with a $\pi \mu$ secondary vertex. Also shown is the background from hadrons misidentified as leptons (BKG 1) and from random combinatoric background (BKG 2); b) shows the same distributions for $\pi e$ candidates; c) shows the combined $\pi \mu$ and $\pi e$ distributions; d) is the same as a) except that the background from misidentified $K \mu \nu$ and from $D^{\circ} \rightarrow K^{*-} l^{+} \nu$ (BKG 3) is shown; c) shows these two backgrounds for $\pi e$; and $f$ ) shows these two backgrounds for the combined distribution. 

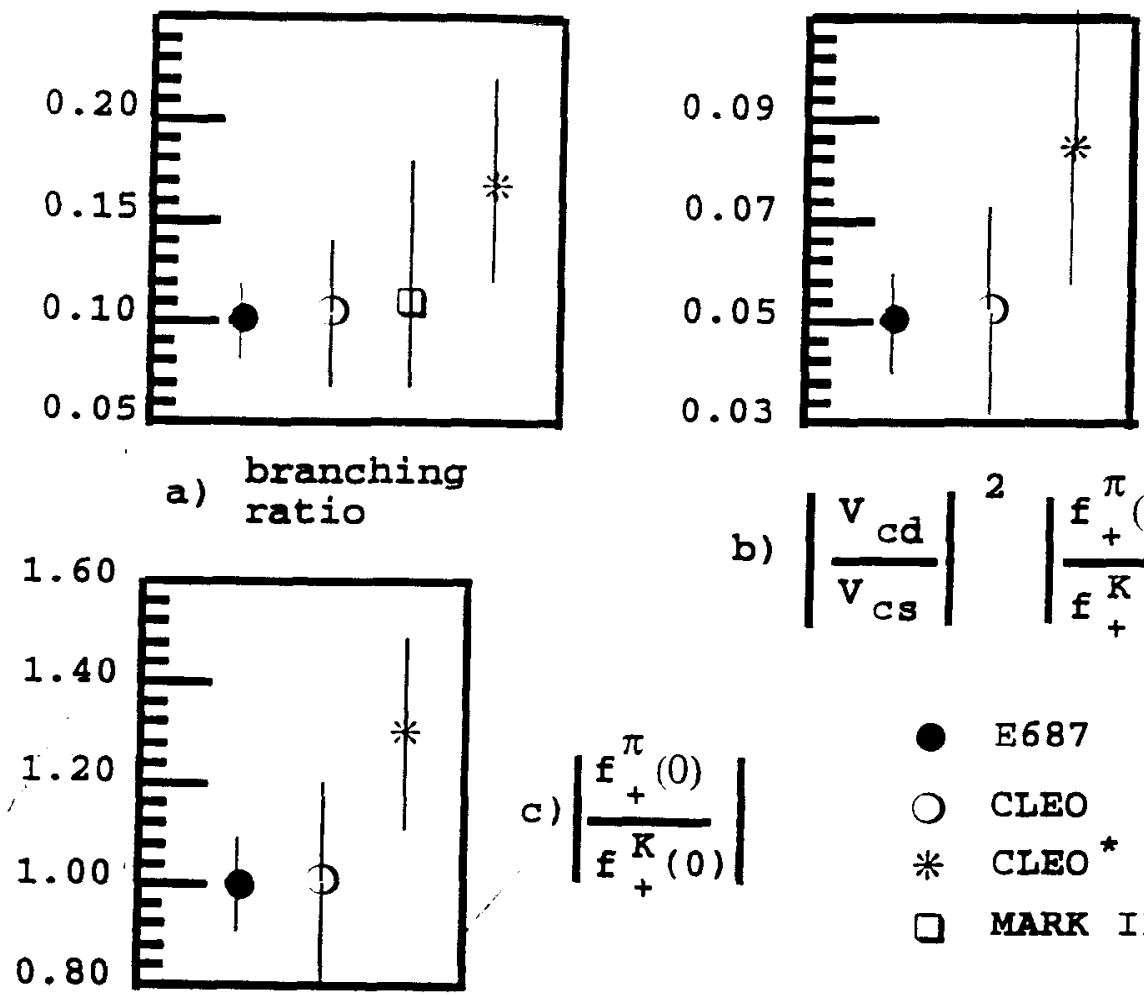

b) $\left|\frac{v_{c d}}{v_{c s}}\right|^{2}\left|\frac{f_{+}^{\pi}(0)}{f_{+}^{K}(0)}\right|^{2}$

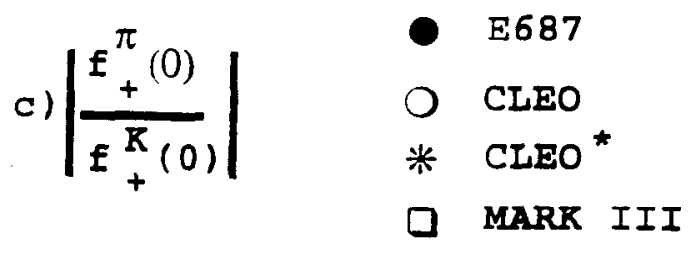

FIGURE 30. Comparison of $D^{\circ} \rightarrow K^{-} l^{+} \nu$ and $D^{\circ} \rightarrow \pi^{-} l^{+} \nu$ : a) Branching ratios; b) CKM elements times form factors at $q^{2}=0$; and c) form factors at $q^{2}=0$ assuming Cabibbo angle values for CKM elements.In this plot, $\mathrm{CLEO}^{*}$ refers instead to the ratio $\frac{D^{+} \rightarrow \pi^{\circ}+l_{\nu}}{D+\rightarrow K^{\circ} l+\nu}$. Data are from reference [37].
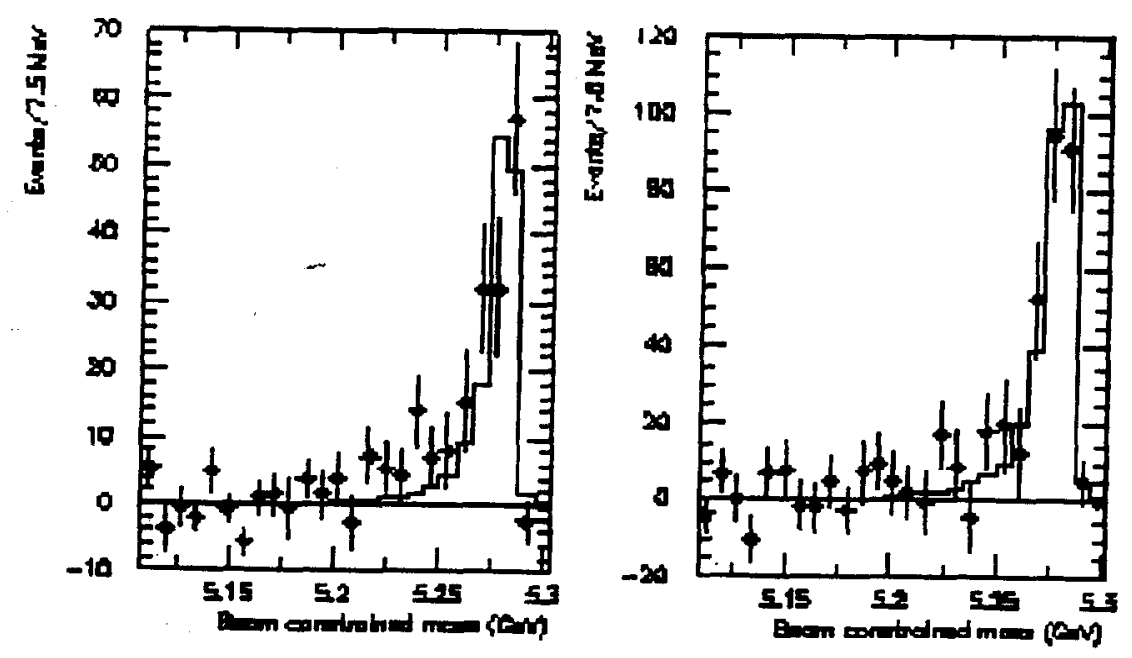

FIGURE 31. Signals for the decay $\bar{B}^{\circ} \rightarrow D^{+} e^{-\bar{\nu}}$ from CLEO 
$\theta_{v}: \quad$ polar angle between $M_{p}$ and $m$ in $M_{v}$ rest frame

$\theta_{l}$ : $\quad$ polar angle between $l$ and $M_{p}$ in $l \nu(\mathrm{W})$ rest frame

$t\left(q^{2}\right)$ : mass of virtual W $\left(M^{2}(l \nu)\right)$

$\chi$ : angle between the two decay planes height

The differential cross section is given by

$$
\frac{d \Gamma}{d M_{m \pi}^{2} d t d \cos \theta_{v} d \cos \theta_{l}} \propto P S \times B W \times|M|^{2} .
$$

The phase space term, PS, is given by

$$
P S \propto K\left(1-\frac{M_{l}^{2}}{t}\right) ;
$$

where $K$ is the $m \pi$ momentum in the parent meson rest frame. The BreitWigner term, BW, for the vector resonance, $V$, is

$$
B W=\frac{\Gamma M_{V}}{\left(M_{m \pi}^{2}-M_{V}^{2}\right)^{2}+M_{V}^{2} \Gamma^{2}}
$$

The matrix element squared $|M|^{2}$ is given by

$$
\begin{aligned}
|M|^{2}= & t\left(1-M_{l}^{2} / t\right) \times \\
& \sin ^{2} \theta_{v}\left[\left(1+\cos \theta_{l}\right)^{2}\left|H_{+}(t)\right|^{2}-\left(1-\cos \theta_{l}\right)^{2}\left|H_{-}(t)\right|^{2}\right. \\
& +4 \cos ^{2} \theta_{v} \sin ^{2} \theta_{l}\left|H_{o}(t)\right|^{2} \\
& +\frac{M_{l}^{2}}{t} \times[\text { lepton mass terms }]
\end{aligned}
$$

The quantities $H_{+}, H_{-}$, and $H_{0}$ are the positive and negative helicity form factors and the longitudinal form factor respectively.

In the limit of $M_{l} \rightarrow 0$, these form factors are related as follows:

$$
\begin{aligned}
H_{ \pm} & =\alpha A_{1}(t) \mp \beta V(t) \\
-\quad h_{0} & =\delta A_{1}(t)-\epsilon A_{2}(t)
\end{aligned}
$$

where the functions $\alpha, \beta, \delta$, and $\epsilon$ are functions of $t, M_{m \pi}$, and $\mathrm{K}$.

Given the level of statistics of current measurements, it is conventional to parameterize the form factors by the pole approximation described above and to express the result as two form factor ratios at $q^{2}=0$ :

$$
R_{v}=\frac{V(0)}{A_{1}(0)}, R_{2}=\frac{A_{2}(0)}{A_{1}(0)}
$$

Applying the same argument we used above, the relevant poles are expected to be the $D^{*}$ and the $D_{s}^{* * \prime}$ 's so it is conventional to take $M_{V}=2.1, M_{A_{1}}=2.5$, and $M_{A_{2}}=2.5 \mathrm{GeV} / \mathrm{c}^{2}$. 


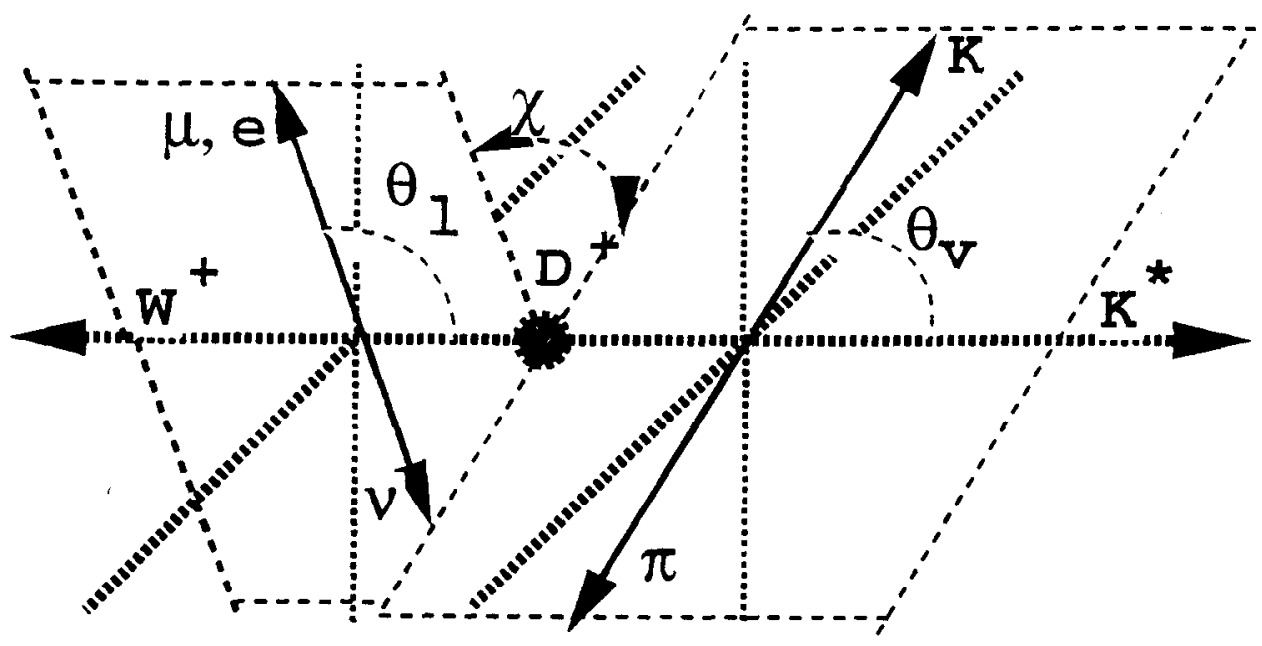

FIGURE 32. Angles used in the description of the semileptonic decay of a pseudoscaler meson to a vector meson.

$$
1 \text { The Decay } D^{+} \rightarrow \bar{K}^{* o}(890) \mu^{+} \nu
$$

Figure 33 shows the $K^{*}$ signal obtained from events with a $K \pi \mu$ detached vertex in photoproduction experiment E687 [40]. The signal appears as a strong $K^{*}(890)$ peak in the invariant mass distribution of the $K^{-} \pi^{+}$from a downstream three body $K^{-} \pi^{+} \mu$ vertex. The world data [41] on the quantities $R_{v}, R_{2}, \Gamma_{L} / \Gamma_{T}{ }^{6}$, and the branching fraction of $K^{* 0} \mu \nu$ relative to $K \mu \nu$ are shown in figure 34 .

$$
2 \text { The Decay } \bar{B}^{\circ} \rightarrow D^{*+} l^{-} \bar{\nu}
$$

This decay is similar to $D^{+} \rightarrow K^{* 0} l^{+} \nu . V_{c b}$ can be extracted from it. So far, this has been done by using the branching fractions. The data [42] on the branching fraction to this mode is shown in table 17. To extract the value of $V_{c b}$ one must use various models of the form factors. The results for $V_{c b}$ using various models are strown in table 18 [43].

\section{Heavy Quark Effective Theory (HQET) and Form Factors}

Above, we discussed Heavy Quark Symmetry in connection with the level spacing of excited charm and bottom mesons. This symmetry can also be applied to hadronic form factors and it results in relationships among the

6) This is the ratio of the longitudinal to transverse polarization. See reference [32] for its relation to the form factors. 

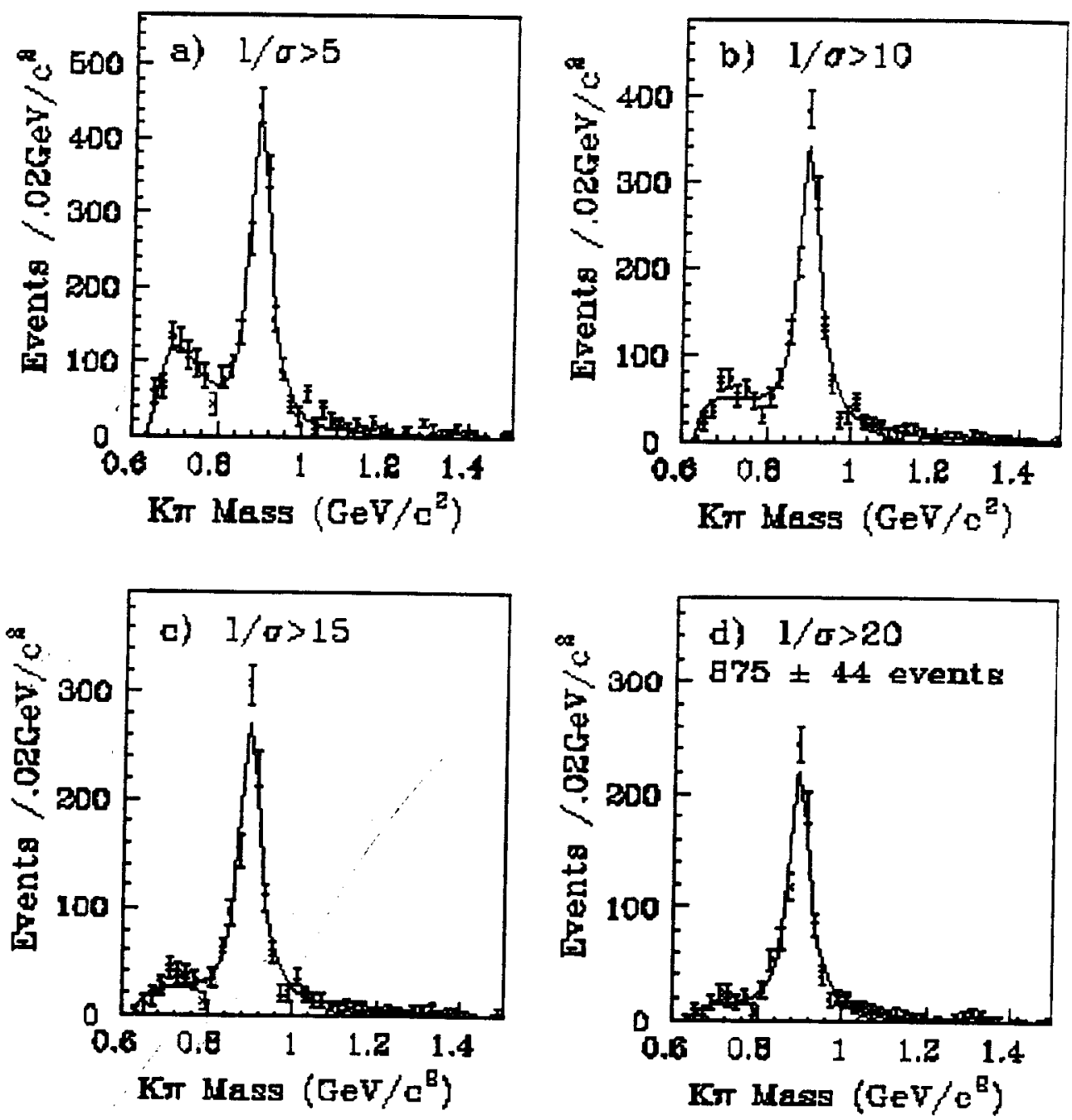

FIGURE 33. The $K^{-} \pi^{+}$distribution showing a strong $\bar{K}^{* 0}$ signal from the decay $D^{+} \rightarrow \bar{K}^{* 0} \mu^{+} \nu$ from E687. a), b), c), and d) show the distribution for a significance of detachment', $\frac{1}{\sigma_{1}}$, of $5,10,15$, and 20 , respectively. The last distribution, d), shows very little background.

TABLE 17. Branching fractions for $B \rightarrow D^{*+1-} \nu(\%)$

\begin{tabular}{|c|l|}
\hline \hline Experiment & $\mathrm{B}(\%)$ \\
\hline CLEO & $4.1 \pm 0.5 \pm 0.7$ \\
ARGUS & $4.7 \pm 0.6 \pm 0.6$ \\
CLEO II & $4.50 \pm 0.44 \pm 0.44$ \\
ALEPH & $5.18 \pm 0.30 \pm 0.62$ \\
DELPHI & $5.47 \pm 0.16 \pm 0.67$ \\
Average & $4.90 \pm 0.35$ \\
& \\
\hline
\end{tabular}



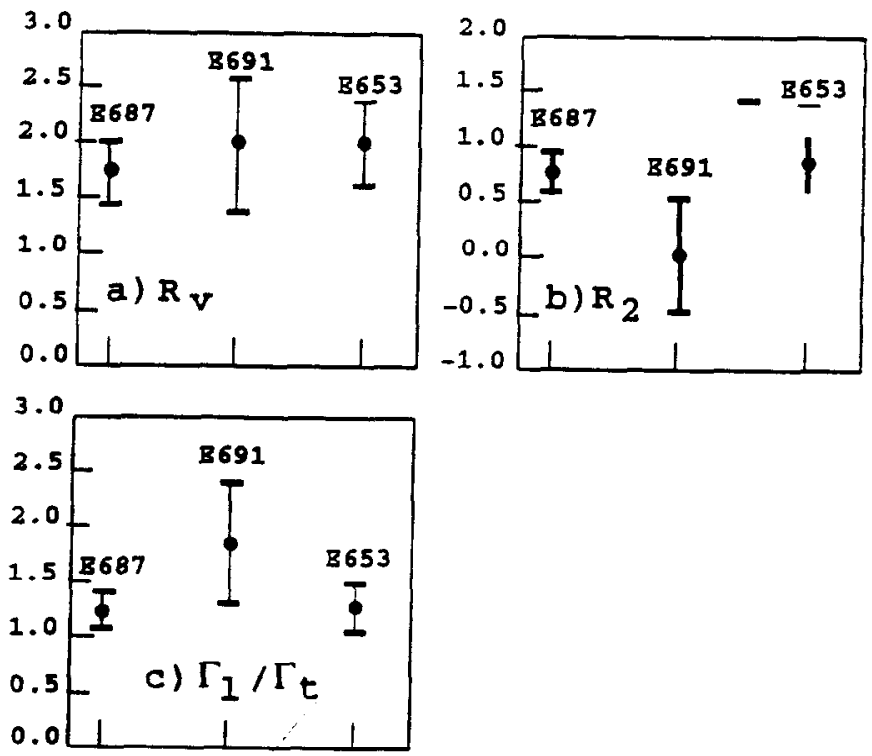

FIGURE 34. Results for the decay $D^{+} \rightarrow \bar{K}^{* 0} \mu \nu$ : a) $R_{v}$; b) $R_{2}$; and c) $\frac{\Gamma_{1}}{\Gamma_{1}}$.

TABLE 18. Predictions for $V_{c b}$ using various form factors

\begin{tabular}{|c|c|c|}
\hline \hline Model & $\Gamma\left(B \rightarrow D^{*} l \nu\right)\left(\mathrm{ps}^{-1}\right)$ & $V_{c b}$ \\
\hline ISGW & $25.2\left|V_{c b}\right|^{2}$ & $0.0352 \pm 0.0014$ \\
ISGW II & $24.8\left|V_{c b}\right|^{2}$ & $0.0355 \pm 0.0014$ \\
KS & $25.7\left|V_{c b}\right|^{2}$ & $0.0348 \pm 0.0014$ \\
WBS & $21.9\left|V_{c b}\right|^{2}$ & $0.0378 \pm 0.0015$ \\
Jaus1 & $21.7\left|V_{c b}\right|^{2}$ & $0.0379 \pm 0.0015$ \\
Jaus2 & $21.7\left|V_{c b}\right|^{2}$ & $0.0379 \pm 0.0015$ \\
\hline
\end{tabular}


form factors which reduce the number of unknown functions and simplifies the extraction of the CKM elements.

The following is a brief summary of HQET:

- HQET should be applicable to the b-quark. Application to the $c$-quark is a bit less justified and corrections for symmetry breaking effects are necessary for accurate comparisons between the two flavors.

- Since all spin and flavor related forces go like $\frac{1}{M_{Q}}$, the only force that governs the behavior of the light degrees of freedom in a meson with a heavy quark in it is the (strong) color force. The light quarks are viewed as moving in a static color field.

- In the limit where the mass of the heavy quark goes to infinity, hadronic systems which differ only in the flavor or spin of the heavy quark have the same configuration of their light degrees of freedom.

- One can show that all form factors in 'heavy quark to heavy quark' decays reduce to one 'universal' function which depends only on the invariant four velocity transfer from the heavy quark to its lighter (but still heavy) daughter quark in the decay. This velocity transfer is related to the meson masses (here taken to be for the decay of a $B$-meson to a $D^{*}$, a case which has actually been studied experimentally) and $q^{2}$ as:

$$
y=\frac{M_{B}^{2}+M_{D^{*}}^{2}-q^{2}}{2 M_{B} M_{D^{*}}}
$$

This quantity is large when $q^{2}$ is small and goes to 1 at $q_{\max }^{2}$ which occurs when the $B$ decays to a $D^{*}$ which is at rest in the $B$ rest frame.

- The universal function $\xi(y)$ is not known a priori but may be derived from models.

- In the lowest order $\mathrm{QCD}, \xi(1)=1$, and higher order terms lower this by around $10 \%$.

To see this, consider the decay of a meson containing heavy quark at $q_{\max }^{2}$ shown in figure 35 . The parent meson is initially travelling along with some velocity and its light quark is orbiting around it. We have seen that the heavy quark just acts as a static color source and otherwise does not couple to the light degrees of freedom. Suddenly, the heavy quark turns into a different heavy quark, which is an (almost) equivalent color source. Thus, in the heavy quark limit, the light degrees of freedom are already in the right configuration to form the daughter meson. This picture corresponds to a value of $y=1$, where the value of the universal form factor $\xi(1)=1$ in lowest order.

More generally, the light constituents of a heavy-light system are not affected by the replacement of the heavy quark, $Q\left(\vec{v}_{Q}, \vec{S}\right)$ with another heavy quark 
$Q^{\prime}\left(\vec{v}_{Q^{\prime}}, \vec{S}^{\prime}\right)$ where the velocities of the two quarks are the same: $\vec{v}_{Q}=\vec{v}_{Q^{\prime}}$. The spins do not matter.

This leads to the idea of a single universal form factor, $\xi$, that depends only on $y$. In the case of pseudoscalar to vector semileptonic decays where in the classical formalism presented above there are several form factors, all of them will be related to this one universal function.

While the symmetry predicts the existence of this universal function, it does not tell us what the function is. The function must be obtained from QCDmotivated models or calculations. There are several versions on the market. Some of the functions proposed are

$$
\begin{aligned}
\xi(y) & =1-\rho^{2}(y-1) \\
& =\frac{2}{y+1} \times \exp ^{-\left(2 \rho^{2}-1\right) \frac{y-1}{y+1}} \\
& =\left(\frac{2}{y+1}\right)^{2 \rho^{2}} \\
& =\exp ^{-\rho^{2}(y-1)}
\end{aligned}
$$

The value of $\xi$ at 1 must be corrected for gluon emission and finite mass effects. If these corrections can be brought under control, then the systematic error from theory in the extraction of the CKM element, $V_{c b}$, will be reduced.

Figure 36 shows $\xi(y)$ measured in by the CLEO collaboration [44]. Stone [45] has recently fit the CLEO data to a variety of forms for $\xi$ and has derived the result

$$
\left|V_{c b}\right| \xi(1) \times 10^{-3}=36.3 \pm 1.6 \pm 1.0
$$

giving a value of

$$
\left|V_{c b}\right|=0.0397 \pm 0.002 \pm 0.0017
$$

This result is slightly higher than previous results.

\section{Charmless Semileptonic $B$ Decays}

Semileptonic $B$ decays which produce non-charmed mesons are the result of the $b$-quark transforming to a $u$-quark and a $W^{-}$. The amplitude is proportional to the CKM parameter $V_{u b}$. CLEO [46] has studied the decays

$$
\begin{aligned}
& B^{-} \rightarrow\left(\pi^{\circ}, \rho^{\circ}, \omega^{\circ}\right)-l \nu ; \text { and } \\
& \bar{B}^{\circ} \rightarrow\left(\pi^{+}, \rho^{+}\right)-l \nu
\end{aligned}
$$

To get a clean signal, they reconstruct the energy and momentum of the $\nu$ by using their knowledge of the total energy and by exploiting their excellent 


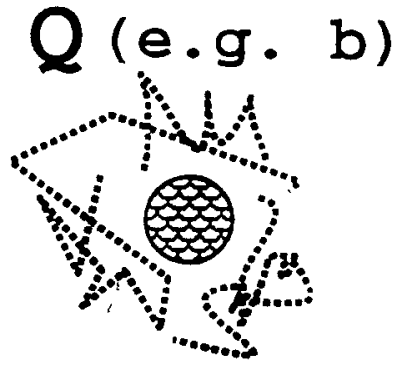

(a)

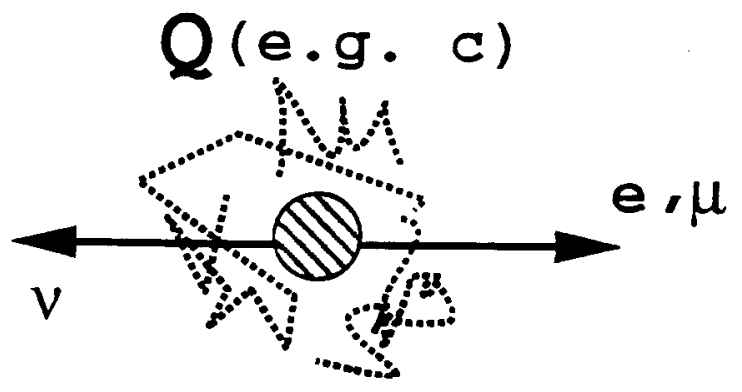

(b)

FIGURE 35. Decay of a meson containing a heavy quark

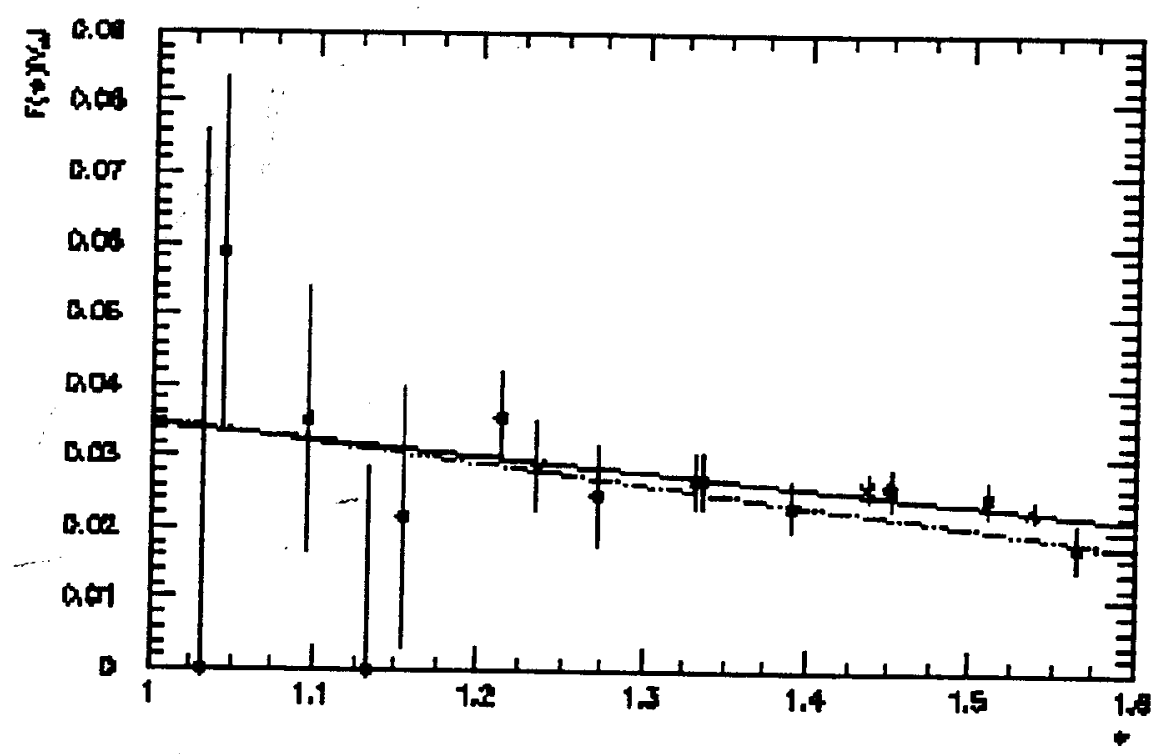

FIGURE 36. Form Factor for $\bar{B}^{\circ} \rightarrow D^{+} e^{-} \bar{\nu}$ plotted vs the Isgur-Wise variable. 


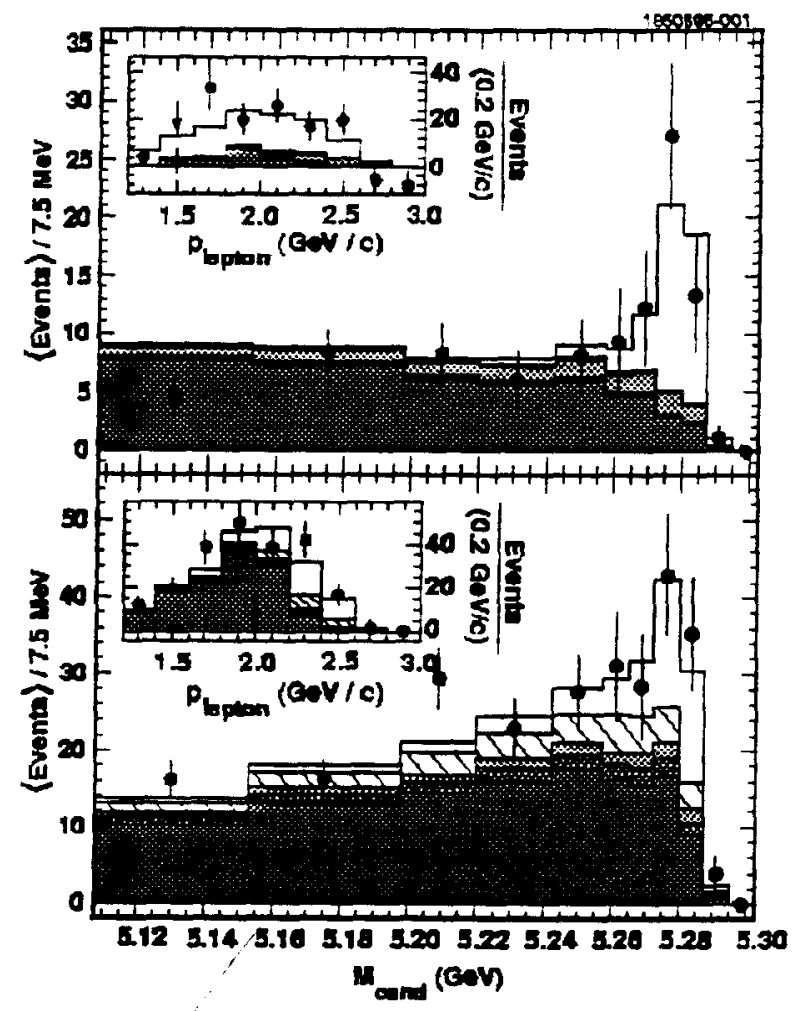

FIGURE 37. Evidence for $B \rightarrow \pi e \nu$ and $B \rightarrow \rho(\omega) e \nu$. The $B$ candidate mass distributions, $M_{\text {eand }}$, for the sum of the scalar $\pi^{+} l \nu$ and $\pi^{\circ} l \nu$ (top) and the vector modes ( $\rho$ and $\omega$ ) (bottom). The points are the data after continuum and fake background subtractions. The unshaded histogram is the signal while the dark shaded area shows the $b \rightarrow c X$ background estimate, the cross hatched area the estimated $b \rightarrow u l \nu$ feedown. For the $\pi$ (vector) modes, the light-shaded and hatched histograms are $\pi \rightarrow \pi$ (vector $\rightarrow$ vector) and vector $\rightarrow \pi(\pi$ $\rightarrow$ vector) crossfeed, respectively. The insets show the lepton momentum spectra for the events in the $B$ mass peak (the arrows indicate the momentum cuts).

angular coverage to get the magnitude and direction of the missing energy in the event.

The experimental evidence for the observation by CLEO [46] of exclusive charmless semileptonic decay is given in figure 37. A rather complicated background subtraction is required to prove that the $\pi \pi$ system really is dominated by the $\rho$. Results for $V_{u b}$ using various models for the form factor are given in table 19 taken from reference [39].

\section{E Leptonic Decays}

Figure 38 shows an example of the purely leptonic decay of a pseudoscalar meson. The decay rate is: 
TABLE 19. Values of $V_{u b} / V_{c b}$ from exclusive $\pi l \nu$ and $\rho l \nu$ analyses combined and taking $V_{c b}=0.0381$

\begin{tabular}{|c|c|}
\hline \hline Model & $V_{u b} / V_{c b}$ \\
\hline ISGW II & $0.089_{-0.011}^{+0.010}$ \\
WBS & $0.076 \pm 0.010$ \\
KS & $0.058_{-0.008}^{+0.006}$ \\
Melnikov & $0.105_{-0.019}^{+0.018}$ \\
\hline \hline
\end{tabular}

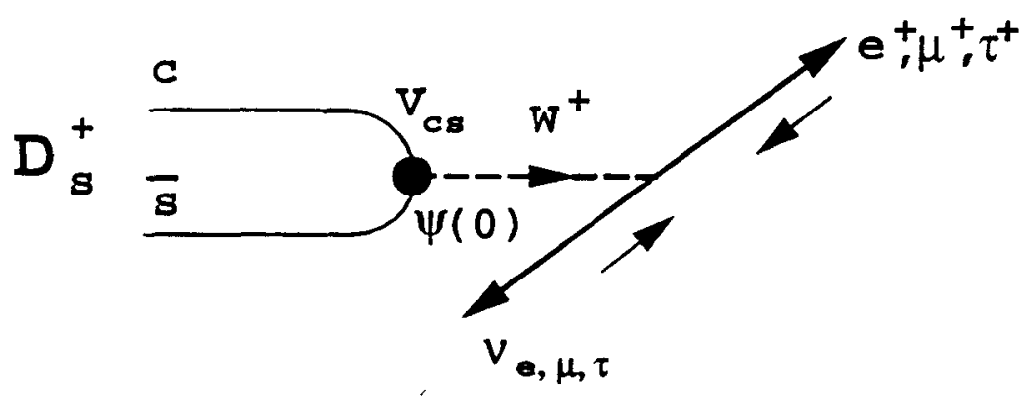

FIGURE 38. Purely leptonic decay of $D_{s}$ meson

$$
\Gamma=\frac{G_{F}^{2}\left|V_{Q_{q}}\right|^{2} f_{X}^{2}}{8 \pi} \times m_{X} m_{l}^{2}\left(1-\frac{m_{l}^{2}}{m_{X}^{2}}\right)^{2}
$$

The 'decay constant', $f_{X}$, gives the probability that the two valence quarks in the parent meson, ' $X$ ', are close enough to experience the short range weak interaction and is proportional to the wave function squared at the origin.

There are several points to note. First is the dependence on the final state lepton mass squared. This is a result of helicity suppression. The neutrino forces the positron to be mainly in a helicity configuration that does not couple to the $W$. The positron then can couple to the $W$ only through its 'small' helicity component, leading to the suppression. The heavier $\mu$ and $\tau$ have larger 'small' components and so have larger leptonic widths. Thus, decays to heaviest lepton, the $\tau$, are heavily favored and decays to positrons are very rare. This is true even though there is some suppression for decay to heavy quarks coming from the last term. Second, since the purely leptonic decay is proportional to the mass of the parent state and the total decay rate goes roughly like the fifth power, the branching fraction for purely leptonic decays will be very tiny for mesons containing heavy quarks.

The reason for interest in this decay is that it provides a relatively direct and clean measure of $f_{X}$. The quantity $f_{B}$ is needed to extract the CKM element $V_{t d}$ from a measurement of $B^{\circ}$ mixing, which will be discussed briefly below. The leptonic $B_{u}$ decay is nearly impossible to observe because it de- 
pends on $V_{u b}$ and because leptonic decays in general are suppressed relative to other decays for heavy quarks as explained above. For now, we must depend on a theoretical calculation. There is a large variation in the results of the various calculations. Any information that would give us confidence in some of the theoretical results would help reduce our uncertainty in this important quantity and in our ability to use theory to extract $V_{\text {td }}$. For that, we must look to charm. There has recently been an observation [47] of the decay $D_{s} \rightarrow \mu \nu$. The resulting value for $f_{D}$, is:

$$
f_{D_{0}}=344 \pm 37 \pm 52 \pm 42 \mathrm{MeV}
$$

where the first two errors are the statistical and systematic errors from the measurement and the third uncertainty is from the error on the absolute branching fraction of $D, \rightarrow \phi \pi$ which is used to convert the signal to a width. This is to be compared with the value of Aoki [48] et al.:

$$
f_{D_{\mathrm{s}}}=232 \pm 45 \pm 20 \pm 48 \mathrm{MeV}
$$

Theoretical predictions have been made using lattice gauge calculations, potential models, sum rules, and other methods which involve using both experimental and theoretical inputs. Typical results lie in the range from 200 to $300 \mathrm{MeV}$.

\section{RARE DECAYS}

While this topic is far too broad and complex to receive a complete treatment in these lectures, I do want to illustrate briefly why 'rare processes' - by which I mean those involving loops or box diagrams at the quark level - play a much larger role in $B$ decays than in charm decays.

The CKM matrix has been written by Wolfenstein [49] in the following form:

$$
V=\left(\begin{array}{ccc|c}
u & c & t & \\
\hline 1-\lambda^{2} / 2 & \lambda & A \lambda^{3}[\rho-i \eta] & d \\
-\lambda & 1-\lambda^{2} / 2 & A \lambda^{2} & s \\
A \lambda^{3}(1-\rho-i \eta) & -A \lambda^{2} & 1 & b
\end{array}\right)
$$

The quantity $\lambda$ is the sine of the normal Cabibbo angle. Since its value is approximately 0.22 , and since $A, \rho$, and $\eta$ are all known to be less than 1.0 [50], it is clear that mixing between quarks of different generations is small. This fact, combined with the observed value of the quark masses, leads to the conclusion that rare decays and mixing, as defined above, are much smaller for charmed particles than for bottom particles within the Standard Model.

Figure 39 shows the box diagrams responsible for flavor mixing and Penguin (loop) diagrams which are responsible for a flavor changing neutral currents for the case of bottom and charm. The expression for $B$-mixing is 


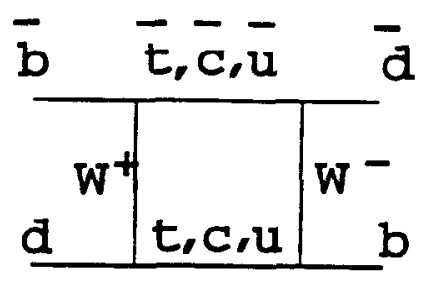

(a)

d

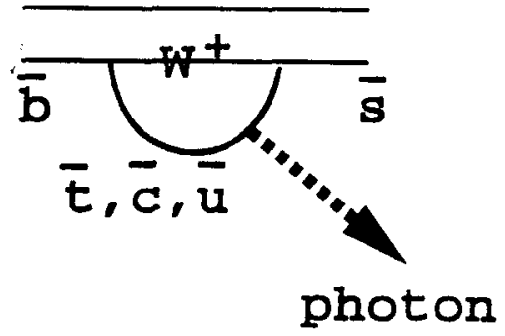

or

gluon

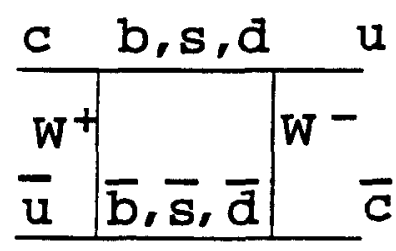

(b)

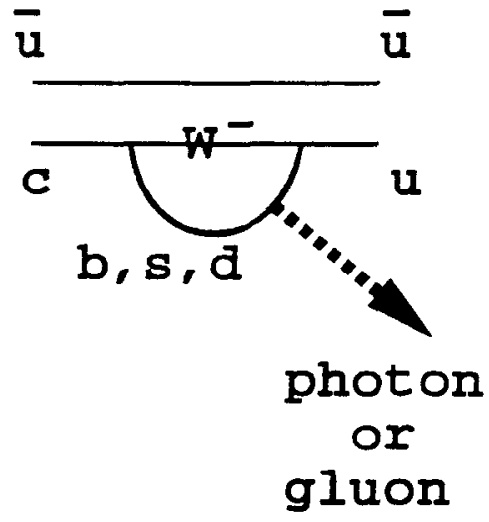

(d)

FIGURE 39. Rare processes: a) $B^{\circ}$ mixing; b) $D^{\circ}$ mixing; c) A penguin diagram for $B^{\circ} \rightarrow K^{* 0} \gamma ;$ and d) A penguin diagram for $D^{\circ} \rightarrow \rho^{\circ} \gamma$.

$$
x_{B}=\frac{\Delta M_{B}}{\Gamma_{B}}=\frac{G_{F}^{2}}{6 \pi^{2}} \tau_{B} B_{B} f_{B}^{2} m_{B} m_{t}^{2}\left|V_{t b}^{*} V_{t d}\right|^{2} F(x) \eta_{Q C D}
$$

where $x=\frac{m_{t}^{2}}{M_{w}^{2}} . B$-mixing is a large effect and has been extensively studied.

It can be seen that the corresponding expression for $D^{\circ}$ mixing will depend on the masses of the objects in the box diagram and the CKM elements as:

$$
x_{D}=\frac{\Delta M_{D}}{\Gamma_{D}} \propto\left|V_{c b}^{*} V_{u b}\right|^{2} m_{b}^{2}
$$

and the ratio is

$$
\frac{x_{D}}{x_{B}} \sim \lambda^{4} \frac{M_{b}^{2}}{M_{t}^{2}}
$$

which is much smaller than $B$-mixing because of the appearance of small CKM elements and because the $b$-quark in the box is so much lighter than the $t$ quark which dominates the $B$-mixing. In fact, the CKM suppression is so strong that the $s$-quark may be the dominant short range contributor to $D$ mixing. So-called long range effects, that is hadrons appearing in the diagram in place of the box of quarks, may increase the mixing by up to an order of 
magnitude but it is still far below the level of $B$-mixing and has never been observed [51].

For similar reasons, penguin (loop) diagrams appear at the level of $10^{-4} \%$ of $B$ decays and have been observed [52], but loop diagrams have not been observed in $D$ decays and SM predictions are that they are very small and unlikely to be observed [53]. For example, the SM prediction for the branching fraction for $D^{\circ} \rightarrow \rho^{\circ} \gamma$ is of order of $10^{-17}$ ! However, long distance contributions may increase this by a large factor.

$\mathrm{CP}$ violating asymmetries in weak decays are expected to arise from the interference between direct decays and flavor mixing followed by decays into the same states [54]. Since $D$-mixing is so small that it has yet to be observed and is predicted to lie well below existing limits, it is clear that the $B$ system is a much more promising one for studies of $C P$ violation. Another way to look at this is that the CKM elements that carry the weak phase and therefore are responsible for $C P$ violation are the ones that involve third generation quarks. Since the $b$-quark is itself a third generation quark, it is strongly connected to $\mathrm{CP}$ violation. The $c$-quark is not a third generation quark and is therefore only weakly connected.

$B$-decays offer a good opportunity to study rare decays which occur within the context of the Standard Model. The outlook for studying these processes for charm is bleak if the only mechanisms by which they can occur are Standard Model processes. Let me end this paper by reminding everyone that, while the $B$ system is very exciting because it allows one to address many fundamental issues, the charm system should not be neglected. If mixing, penguin processes, or CP violation show up at a level significantly higher than predicted by the Standard Model, it might be an indication of new physics!

\section{CONCLUSION}

In these lectures, we have shown that in some cases particles with charm and bottom quarks are very similar - in their spectroscopy and semileptonic decays - and in other cases are very different - in their lifetime patterns and likelihood of undergoing rare decays. In all cases, the Standard Model offers a sound explanation for the observed behavior.

\section{Acknowledgements:}

I would like to thank the conference organizers for their hospitality and for giving me the opportunity to participate as both a student and a lecturer in the VII Mexican School of Particles and Fields. I want to thank Sheldon Stone and James E. Wiss from whose lectures at the NATO Advanced Institute and the SLAC Summer School I borrowed copiously. I also want to thank all my colleagues on the E687 and FOCUS/E831 experiments and the whole B Study Group at Fermilab who have taught me much of the physics presented here. Finally, I would like to thank my colleague Hector Mendez 
for his help and 'gentle' encouragement in getting me to write up these lectures. This article was written at and the work supported by the Fermi National Accelerator Laboratory, which is operated by Universities Research Association, Inc., under contract DE-AC02-76CH03000 with the U.S. Department of Energy.

\section{REFERENCES}

1. See, for example, Chris Quigg, Gauge Theories of the Strong, Weak, and Electromagnetic Interactions, Frontiers in Physics, Benjamin-Cummings Press, Menio Park (1983).

2. M. Kobayashi and K. Maskawa, Prog. Theor. Phys. 49,652(1973).

3. Review of Particle Properties, Phys. Rev. D 50, p1173-1826(1994). See page 1319.

4. See reference [3], page 1321.

5. See reference [3], page 1782 .

6: J.G. Korner, "Charm Baryons, Theory and Experiment", Ann. Rev. Nucl. Part. Sci. 41, 511-45(1991).

7. Frabetti et al., Phys. Lett. 'B 323, 459(1994).

8. L. Gibbons et al., "Observation of an Excited Charmed Baryon Decaying into $\Xi_{c}^{0} \pi^{+}$", Phys.Rev. Lett. $77,810(1996)$ and P. Avery et al., "Observation of a Narrow State Decaying into $\Xi_{c}^{+} \pi^{-"}$, Phys. Rev. Lett. 75, 4364(1995).

9. G. Brandenburg et al., "Observation of Two Excited Charmed Baryons Decaying into $\Lambda_{c}^{+} \pi^{ \pm "}$, clns $96 / 1427$, cleo 96-113(1996).

10. K.W. Edwards et al., "Observation of Excited Charmed Baryon States Decaying to $\Lambda_{c}^{+} \pi^{+} \pi^{-"}$, Phys. Rev. Lett. 74, 3331(1995).

11. F. Abe et al., "Observation of $\Lambda_{b}^{\circ} \rightarrow J / \psi \Lambda$ at the Fermilab Proton-Antiproton Collider", Phys. Rev. D 55, 1142 (1997)

12. CDF resuit is reference [11]; C. Albajar et al., Phys. Lett. B 273, 540(1991); P. Abreu et al., Phys. Lett. B 374, 351(1996); and D. Buskulic et al., Phys. Lett. $B$ 380, 442(1996).

13. Albrecht H. et al.,-ARGUS Collaboration, Phys. Rev. Lett. 56, 549 (1986).

14. See John Bartelt and Shekhar Shukla, "Charmed Meson Spectroscopy", Annu. Rev. Nucl. Part. Sci. 45, 133-161(1995).

15. N. Isgur and M. Wise, "Heavy Quark Symmetry", in B Decays revised second edition, p231-282, edited by Sheldon Stone, World Scientific (1994).

16. Frabetti et al., "Measurement Of The Masses And Widths Of $\mathrm{L}=1$ Charm Mesons", Phys. Rev. Lett. 72, 324(1994).

17. See reference [14] references $18,21,20,2216,17,19$ for $D_{2}^{* 0,+}$; references 5,20 , 16,22 , and 19 for $D_{1}^{o,+}$; references $5,20,25,19$, and 28 for $D_{s 1}^{+}$; and reference 23 for $D_{s 2}^{*+}$.

18. See John Bartelt and Shekhar Shukla, "Charmed Meson Spectroscopy", Annu. Rev. Nucl. Part. Sci. 45, 133-161(1995). 
19. E. Eichten, C.T. Hill, and C. Quigg, "Properties of Orbitally Excited HeavyLight Mesons", Phys. Rev. Lett. 71, 4116(1993).

20. D. Buskulic et al., Z. Phys. C 69, 393(1996).

21. P. Abreu et al., Phys. Lett. B 345, 598(1995).

22. R. Akers et al., Z. Phys. C 66, 19(1995).

23. M. Feindt and O. Podobrin, DELPHI 96-93 CONF-22, 25 June 1996 (paper submitted to ICHEP96).

24. C. Quigg and E. Eichten, "Mesons with Beauty Charm" Phys. Rev. D 49, 5845(1994).

25. R.M. Barnett et al., Phys. Rev. D54, 1 (1996).

26. M. Wirbel. B. Stech, and M. Bauer, Z. Phys. C 29, 637(1985), M. Bauer and M. Wirbel, $Z$. Phys. C 42, 671(1989).

27. T.E. Browder, K. Honscheid, and D. Pedrini, "Nonleptonic Decays and Lifetimes of Charm and Beauty Particles" in Annual Review of Nuclear and Particle Science Vol. 46, p458-461(1996).

28. K. Honscheid, "Hadronic Decays of $B$ Mesons", in the proceedings of The Advanced Study Conference on Heavy Flavors, p 61-76, Pavia (Italy), 1993.

29. B. Guberina, R Ruckl, and J. Trampetic, "Charmed Baryon Lifetime Differences", $Z$. Phys. C 33, 297-305(1986).

30. a) Frabetti et al., "Measurement of the Mass and Lifetime of the $\Xi_{c}^{+n}$, Phys. Rev. Lett. 70, 1381(1993); b) Frabetti et al., "A Measurement of the $\Lambda_{c}^{+}$Lifetime", Phys. Rev. Lett. 70, 1755(1993); c) Frabetti et al., "Measurement of the Lifetime of the $\Xi_{c}^{o n ;}$, Phys. Rev. Lett. 70, 2058(1993); and d)Frabetti et al, "Measurement of the $\Omega_{c}^{o}$ Lifetime", Phys. Lett. B 357, 67(1995).

31. F. Abe et al., Phys. Rev Lett. 77, 1439(1996); D. Buskulic et al., Phys. Lett. B 357, 685(1995); P. Abreu et al., Z. Phys. C 71, 199 (1996); and R. Akers et al., Z. Phys. C. 69, 195(1966).

32. An excellent reference is Gilman, F.J. and Singleton, R.L., "Analysis of semileptonic decays of mesons containing heavy quarks", Phys. Rev. D 41, 142(1990).

33. A. Bean et al., "Measurements of Exclusive Semileptonic Decays of $D$ Mesons", Phys. Lett. B 317, 647(1993).

34. Frabetti et al, "Analysis of the Decay Mode $D^{\circ} \rightarrow K^{-} \mu^{+} \nu^{\prime}$, Phys. Lett. B 364, 127(1995).

35. A. Bean et al., "Measurements of Exclusive Semileptonic Decays of $D$ Mesons", Phys. Lett. $B$ 317, 647(1993).

36." Frabetti et al., "Analysis of the Cabibbo Supressed Decay $D^{\circ} \rightarrow \pi^{-} l \nu$ ", Phys. Lett. B 382, 312(1996).

37. F. Butler et al., "Measurement of the Ratio of Branching Fractions $\left(D^{0} \rightarrow\right.$ $\left.\pi^{-} e^{+} \nu_{e}\right) /\left(D^{0} \rightarrow K^{-} e^{+} \nu_{e}\right) "$, Phys. Rev. D 52, 2656 (1995) and M.S. Alam et al., "Measurement of the Ratio $\mathcal{B}\left(D^{+} \rightarrow \pi^{0} l^{+} \nu\right) / \mathcal{B}\left(D^{+} \rightarrow \bar{K}^{0} l^{+} \nu\right)$ ", Phys. Rev. Lett. 71,1311 (1993).

38. T. Bergfeld et al., "Measurement of $B\left(\bar{B}^{\circ} \rightarrow D^{+} l^{-} \bar{\nu}\right)$ and Extraction of $V_{c b}$ ", CLEO-CONF 96-3, ICHEP-96 PA05-78(1996).

39. S. Stone, "Prospects for $B$ Physics in the Next Decade", Presented at NATO Advanced Study Institute on Techniques and Concepts of High Energy Physics, 
Virgin Islands, July 1996. Syracuse Preprint HEPSY 96-01, October 1996.

40. Frabetti et al., "Analysis of the Decay Mode $D^{+} \rightarrow K^{* 0^{-}}+\nu^{\prime}$, Phys. Lett. $B$ 307, 262(1993).

41. E687 data are from reference [40]; E691 Collaboration, J.C. Anjos et al., Phys. Rev. Lett. 65, 2630(1990); and E653 Collaboration, K. Kodama et al., Phys. Lett. B 274, 246(1992).

42. D. Bortolet to et al., Phys. Rev. Lett. 16, 1667(1989) and J.E. Duboscq et. al., "Measurement of the Form Factors for $\bar{B}^{0} \rightarrow D^{*+l}-\bar{\nu}$ ", Phys. Rev. Lett. 76, 3898 (1996).

43. B. Grinstein, N. Isgur and M.B. Wise, Phys. Rev. Lett. 56, 258(1986); N. Isgur, D. Scora, B. Grinstein, and M.B. Wise, Phys. Rev. D 39, 799(1989); W. Jaus, Phys. Rev. D 41, 3394(1990); M. Wirbel, B. Stech, and M. Bauer, Z. Phys. $C$ 29, 637(1985); and M. Bauer and M. Wirbel, $Z$. Phys. $C$ 42, 671(1989); and J.G. Korner and G.A. Schuler, Z. Phys. C 38, 511(1988). ibid,(erratum)C 41, 690(1989).

44. see reference [39], p $16-20$.

45. reference [39], p 19.

46. J.P. Alexander et al., "First Measurement of the $B \rightarrow \pi l \nu$ and $B \rightarrow \rho(\omega) l \nu$ Branching Fractions", Phys. Rev. Lett. 77, 5000(1996).

47. D. Acosta et al., "First Measurement of $\Gamma\left(D_{a}^{+} \rightarrow \mu^{+} \nu\right) / \Gamma\left(D_{a}^{+} \rightarrow \phi \pi^{+}\right)$", Phys. Rev. $D$ 49, 5690(1994).

48. S. Aoki et al., Progress of Theoretical Physics 89, 131(1993).

49. L. Wolfenstein, Phys. Rev. Lett. 51, 1945 (1983).

50. B Decays, J. L. Rosner, "The Cabibbo-Kobayashi-Masakawa Matrix", in B Decays, revised second edition, edited by Sheldon Stone, World Scientific, Singapore(1994).

51. Gustavo Burdman, "Charm Mixing and CP Violation in the Standard Model", Proceedings of the CHARM2000 Workshop, Fermilab, June 7-9, 1994, Editors: Daniel M. Kaplan and Simon Kwan and ibid. Jim Wiss, "Semileptonic Decays, Absolute Branching Ratios and Charm Mixing".

52. R. Ammar et al., "Radiative Penguin Decays of the $B$ Meson", CLEO-CONF 96-6 (1996) and M.S. Alam et al., Phys. Rev. Lett. 74, 288(1995).

53. Sandip Pakvasa, "Charm as a Probe of New Physics", Proceedings of the CHARM2000 Workshop, Fermilab, June 7-9, 1994, Editors: Daniel M. Kaplan and Simon Kwan.

54. see for example, I.I.Bigi, V.A. Khoze, N.G. Uraltsev, and A.I. Sanda, "The Question of CP Noninvariance - As Seen Through The Eyes of Neutral Beauty", in CP VIOLATION, editor C. Jarlskog, World Scientific, Singapore, 1989. 